\title{
Dynamics of the ionization bicone of NGC 1068 probed in mid-infrared with VISIR ${ }^{\star} \star \star \star$
}

\author{
A. Poncelet ${ }^{1,2}, \mathrm{H} \cdot \mathrm{Sol}^{1}$, and G. Perrin ${ }^{2}$ \\ ${ }^{1}$ LUTH, Observatoire de Paris, CNRS, Université Paris Diderot, Place Jules Janssen, 92190 Meudon, France \\ e-mail: anne.poncelet@obspm.fr \\ ${ }^{2}$ LESIA, Observatoire de Paris, 92195 Meudon Cedex, France
}

Received 20 August 2007 / Accepted 6 December 2007

ABSTRACT

\begin{abstract}
Context. We present high-spatial and medium-spectral resolution imaging and spectroscopy of the core of the Seyfert II galaxy NGC 1068, obtained in the $N$-band spectral range with the VLT/VISIR (the VLT Imager and Spectrometer in the InfraRed). Spectra were acquired in the ArIII $8.99 \mu \mathrm{m}$, SIV $10.51 \mu \mathrm{m}$ and NeII $12.81 \mu \mathrm{m}$ filters for two orientations of the long slit $\left(2^{\prime} \times 00^{\prime} 4\right)$ : PA $=-15^{\circ}$ and $90^{\circ}$ oriented $\mathrm{N}$ to $\mathrm{E}$.

Aims. The spatial evolution of the [ArIII] $+[\mathrm{MgVII}],[\mathrm{SIV}]$ and $[\mathrm{NeII}]$ lines are investigated to constrain the gas dynamics inside the narrow line region (NLR), and to deduce the geometry and physics of the ionization cones.

Methods. Spectra are extracted in 0.'381 steps along the two slit positions and we obtain line parameters from fits with Gaussian profiles. The computed line ratios are compared to photoionization models.

Results. Lines are resolved and detected in emission up to $\sim 3^{\prime \prime} \mathrm{NW}$ and $\sim 3^{\prime \prime}$ SE of the core, generally double-peaked. Features trace an outflow with radial velocities up to $10^{3} \mathrm{~km} \mathrm{~s}^{-1}$, well reproduced by a hollow biconical structure associated with the ionization bicone of NGC 1068. It is inclined at $\sim 11^{\circ}$ and the maximum aperture is $\sim 80^{\circ}$, in good agreement with optical spectroscopic studies. Conclusions. The velocity profiles traced in the MIR are interpreted as evidence for a gas outflow along the edges of the ionization bicones. The comparison of line ratios with standard dust-free and dusty, radiation pressure-dominated photoionization models shows that the associated gas metallicity is close to the solar value and points to a relatively weak ionization parameter of a typical value $U_{0}=10^{-2.6}$ at $\mathrm{PA}=-15^{\circ}$ along the ionization bicone.
\end{abstract}

Key words. line: profiles - galaxies: Seyfert - infrared: galaxies - techniques: spectroscopic

\section{Introduction}

NGC 1068 is the archetypal Seyfert 2 galaxy, according to the broad polarized lines observed by Antonucci \& Miller (1985, at the distance of $\left.14.4 \mathrm{Mpc}, 1^{\prime \prime}=72 \mathrm{pc}\right)$. Following the unified scheme of AGNs, this means that the dusty torus is viewed edgeon and is responsible for obscuring the radiative central source and the broad line region (BLR). In the standard model, the aperture of the dusty torus defines the location of the ionization cone, i.e. the region where gas and dust of the BLR and of the narrow line region (NLR) are directly irradiated by the central source. The first mechanism stated to account for observed high degree of ionization was the photoionization (Greenstein \& Schmidt 1964), followed by interpretations in terms of shock ionization between NLR material and the ambient medium (van der Kruit et al. 1972). However, this second class of mechanisms fails to reproduce the strongly ionized coronal lines or the large outflow velocities observed in some objects (Kraemer \& Crenshaw 2000; Cecil et al. 2002). The dusty radiation pressure-dominated photoionization models proposed by Groves et al. (2004b)

\footnotetext{
* Based on open time observations collected with VISIR at the ESO/Paranal MELIPAL telescope.

$\star \star$ Tables 2, 3 and Figs. 12 to 23 are only available in electronic form at http://www . aanda.org
}

succeeded in reproducing many observed Seyfert line ratios (Groves et al. 2004c).

In the case of NGC 1068, the [NeV] and [NII] emission-line observations of Bergeron et al. (1989) and Cecil et al. (1990) respectively, gave the first estimates about the geometry of the ionization cone. Hubble Space Telescope (HST) [OIII] and $\mathrm{H} \beta$ emission-line spectra (Cecil et al. 2002) and HST UV spectroscopy (Groves et al. 2004a) showed that the observed NLR material is prominently excited by the strong radiation field from the central engine, and highlighted some local jet-interstellar medium (ISM) shocks. Crenshaw \& Kraemer (2000) and Das et al. (2006) reproduced these data using a model of biconical outflow. Spectroscopy in the near-infrared (NIR) has been investigated by Oliva et al. (2001) who used the emission properties of the [Fe II] line to propose a tool to investigate the role of shock excitations in AGNs and by Galliano \& Alloin (2002) who traced the evolution of $\mathrm{Br} \gamma$ and $\mathrm{H}_{2}$ emission lines inside the nucleus.

Lutz et al. (2000) made use of ISO/SWS (the Infrared Space Observatory-Short Wavelength Spectrometer) to investigate emission lines in the mid-infrared (MIR) and detected the $[\mathrm{ArIII}]+[\mathrm{MgVII}]$, [SIV] and $[\mathrm{NeII}]$ lines. This is consistent with material in a highly ionized outflow and with lower excitation in the extended NLR. Siebenmorgen et al. (2004) analyzed MIR spectra of several starbursts galaxies or AGNs obtained with both ISO (aperture of $14^{\prime \prime} \times 20^{\prime \prime}$ ) and TIMMI2 (aperture of $3^{\prime \prime}$ ) 
installed on the $3.6 \mathrm{~m}$ telescope at ESO. They did not find PAH emission in the central few arcseconds of AGN, ruling out the presence of a starburst there. High-resolution MIR spectroscopy with Michelle at the Gemini North Telescope and the Long Wavelength Spectrometer at Keck is presented by Mason et al. (2006) and Rhee \& Larkin (2006), respectively. Slits are positioned along bright NLR knots viewed in the VISIR deconvolved images (Galliano et al. 2005). They found no evidence for PAH emission and provide the spatial evolution of the wide $9.7 \mu \mathrm{m}$ silicate absorption feature and of the [ArIII]+[MgVII], [SIV] and [NeII] emission lines across the NLR. Mason et al. (2006) derived a color temperature of dust on the core of $\sim 350 \mathrm{~K}$. This is associated with the dusty torus which has been resolved in interferometry with the VLT/MIDI (Mid-InfrareD Interferometer; Jaffe et al. 2004; Poncelet et al. 2006), showing that it is compact and warm (size $<85$ mas and temperature $\lesssim 360 \mathrm{~K}$ ).

In this paper, we investigate the emission of the [ArIII]+[MgVII] $8.99 \mu \mathrm{m}$, [SIV] $10.51 \mu \mathrm{m}$ and [NeII] $12.81 \mu \mathrm{m}$ emission-lines across the NLR thanks to high spatial and spectral resolution spectroscopic data obtained with VISIR. Data and their processing are presented in Sects. 2 and 3. Results on the spatial evolution of emission lines and the comparisons with photoionization models are detailed in Sect. 4. Results are further discussed in Sect. 5 and general conclusions are given in Sect. 6.

\section{Observations}

\subsection{Images}

Images have been obtained with VISIR at VLT/UT3 (ESO/Paranal, Chile), equipped with a DRS (former Boeing) $256 \times 256$ pixels BIB detector ${ }^{1}$. They were acquired during the nights of 2005 November 15 and 22, have been obtained in standard mode in six narrow-band filters across the $N$-band: PAH1 $(8.59 \mu \mathrm{m}, \Delta \lambda=0.42 \mu \mathrm{m})$, ArIII $(8.99 \mu \mathrm{m}$, $\Delta \lambda=0.14 \mu \mathrm{m}), \operatorname{SIV} 2(10.77 \mu \mathrm{m}, \Delta \lambda=0.19 \mu \mathrm{m})$, PAH2 $(11.25 \mu \mathrm{m}, \Delta \lambda=0.59 \mu \mathrm{m})$, NeII $(12.81 \mu \mathrm{m}$, $\Delta \lambda=0.21 \mu \mathrm{m})$, NeII2 $(13.04 \mu \mathrm{m}, \Delta \lambda=0.22 \mu \mathrm{m})$. The exposure time varied between $16 \mathrm{~ms}$ and $625 \mathrm{~ms}$ depending on the filter used. The pixel scale of images is $0.075^{\prime \prime}$. pixel ${ }^{-1}$. Weather conditions were generally good, with a typical airmass of 1.13 and an average optical seeing estimate of $\sim 0.9^{\prime \prime}$. Standard stars from Cohen et al. (1999) were observed before the observations of NGC 1068, for PSF determination.

Images have been reduced with the standard pipeline for data reduction provided by $\mathrm{ESO}^{2}$. Observations were performed with the standard chopping and nodding techniques in order to suppress atmosphere and telescope background emissions. The chopping frequency was $0.1 \mathrm{~Hz}$ and the chopping throw was 5" in the NS direction. The nodding was set parallel to the chopping. Chopping exposures are subtracted to correct for sky and telescope background, then shifted and co-added. The photometry has not been estimated since the presentation of these images only aims to show the evolution of structures with wavelength, and the major interest of the study is based on the spectroscopy. Images have been deconvolved with a standard maximum likelihood algorithm (100 iterations Varosi \& Landsman 1993). For

\footnotetext{
${ }^{1}$ See the VISIR User Manual available through the ESO website: WwW. eso.org/instruments/visir/doc/

${ }^{2}$ See the VISIR Pipeline User Manual accessible via the ESO ftp site: ftp://ftp.eso.org/pub/dfs/pipelines/visir/ visir-manual-1.0.pdf
}

this, the point spread function (PSF) used is given by the observations of a reference star made before the observations of NGC 1068. They are presented in Fig. 1.

\subsection{Spectroscopy}

Data on the core of NGC 1068 were obtained in mediumspectral resolution $(R \sim 3000)$ spectroscopy with VISIR on the night of 2005 December 16, using three different filters: ArIII $8.99 \mu \mathrm{m}(\Delta \lambda=0.188 \mu \mathrm{m}), \operatorname{SIV} 10.51 \mu \mathrm{m}(\Delta \lambda=0.21 \mu \mathrm{m})$ and NeII $12.81 \mu \mathrm{m}(\Delta \lambda=0.21 \mu \mathrm{m})$. They correspond to two orientations of the long slit $\left(2^{\prime} \times 00^{\prime} 4\right):-15^{\circ}$ and $90^{\circ}$ from $\mathrm{N}$ to $\mathrm{E}$ (slit positions and quadrants used for the extraction of the spectra are superposed on images corresponding to the same filters in Fig. 1). For flux calibrations, a standard star (HD 11353 of spectral type K0III) was observed in the three filters before the observations of NGC 1068 for each slit position. The exposure time for individual spectra was $625 \mathrm{~ms}$. The spatial field of view of one pixel is 0.127 . pixel ${ }^{-1}$.

The airmass was $\sim 1.15$ at the time of observations. Seeing conditions were extremely good at the beginning of observations (optical seeing of $\sim 0$. $^{\prime}$ ) and progressively degraded (up to $1^{\prime \prime} .9$ ). Since observations with the slit at $\mathrm{PA}=-90^{\circ}$ were taken at the end of the run, they suffered more of the less favorable conditions. The chopping frequency was $0.1 \mathrm{~Hz}$ and the chopping throw was $6^{\prime \prime}$ for the calibrator star and $8^{\prime \prime}$ for NGC 1068, along slit orientations. The nodding direction was set parallel, with the offset equal to the chopping throw. Images were co-added in real time to store only two co-added images (one for each chopping position). Due to the nodding, they contain three spectra: one positive and two negative.

\section{Processing of spectroscopic data}

Data were reduced in a standard way by the pipeline developed at the CEA by E. Pantin (Pantin 2007, in prep.). The chopping pairs were subtracted to correct for most of the sky and telescope background. The resulting two dimensional spectra (2D) were then corrected from stripes appearing during the acquisition and from the spectral distortion with a first degree polynomial ${ }^{1}$. They were shifted and added, and then wavelength calibrated by fitting the telluric absorption lines. The source spectra were corrected from the telluric line features during the flux calibration, from the division by the reference star spectra. However, there were some residuals left in the source spectra. These were estimated in regions of the spectra free of source flux and corrected afterwards by subtracting them from the source spectra. Spectra were flux calibrated using the composite spectrum of HD 11353 from templates of Cohen et al. (1999).

According to the diffraction limit of the $8.2 \mathrm{~m}$ VLT telescope across the $N$-band $(0,226,0.264$ and 0.322 in the ArIII, SIV and NeII filters respectively), spectra were spatially extracted in 3 pixel sections (i.e. 0!'381), resulting in fields of view of $0.4 \times 00^{\prime} 381$ (referred to as "quadrants" in the following) for individual spectra. The 3 pixels centered on the maximum of flux density were considered to match the nucleus core (this field is refered to as core hereafter; see Fig. 1).

Error bars on individual spectra were calculated from the addition of the standard deviation of background fluctuations, the photon noise (each being estimated on raw spectra of NGC 1068 and of the calibrator star), and the tabulated error on the composite spectra of the calibrator star from Cohen et al. (1999). Spectra and associated error bars, plotted as a function of the rest wavelength (the redshift of NGC 1068 is $z=0.003786$; 

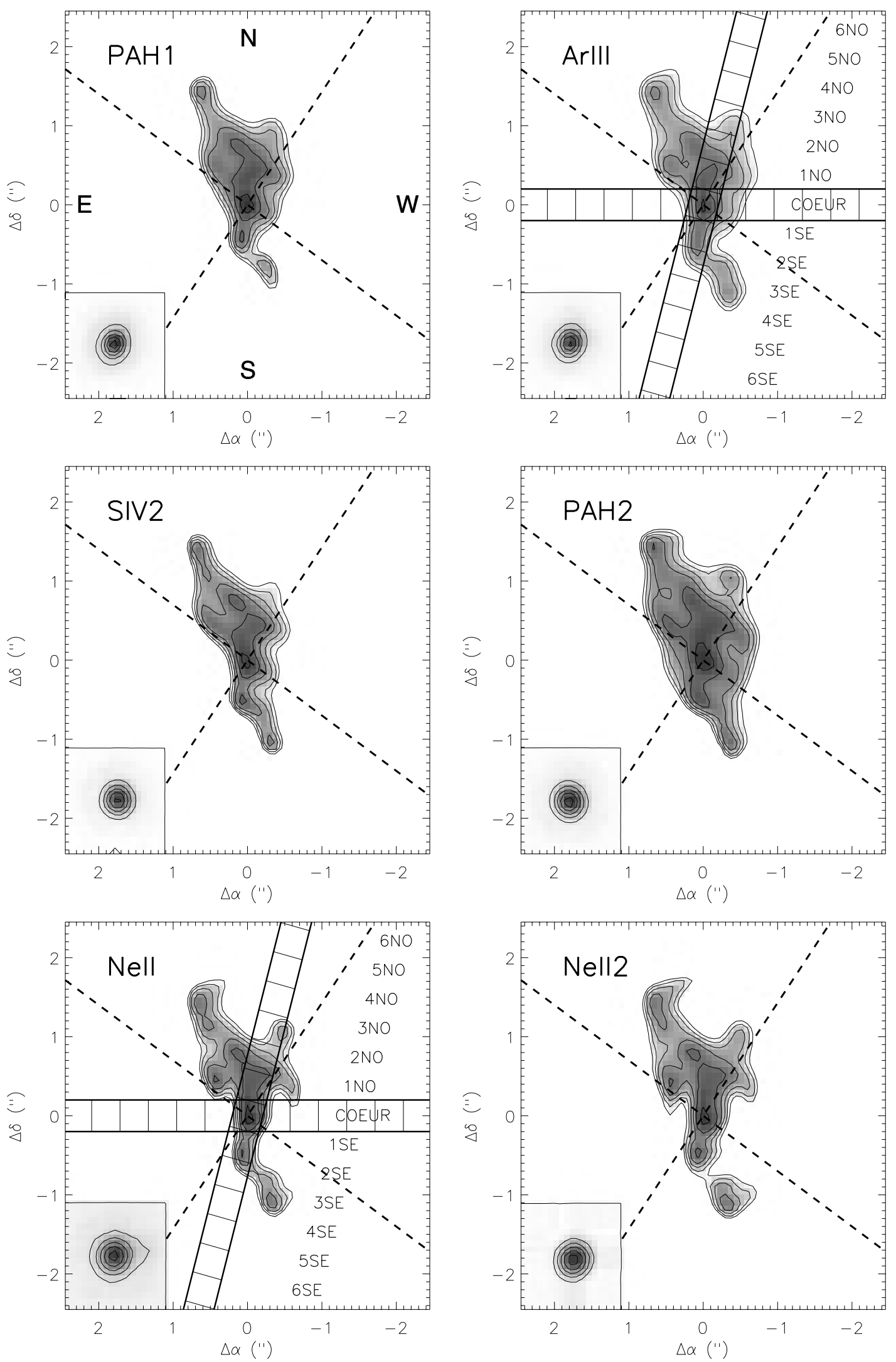

Fig. 1. VISIR images acquired in different narrow band filters: PAH1 $(8.59 \mu \mathrm{m})$, ArIII $(8.99 \mu \mathrm{m}), \operatorname{SIV} 2(10.77 \mu \mathrm{m})$, PAH2 $(11.25 \mu \mathrm{m})$, NeII $(12.81 \mu \mathrm{m})$, NeII2 $(13.04 \mu \mathrm{m})$. Slit positions at PA $=90^{\circ}$ and $-15^{\circ}$ and quadrants associated to 0 '. $^{\prime} 381$ steps of spectra extraction are reported on the image of same filters as ones used in spectroscopy. The two dotted lines represent the ionization cone edges as derived in Sect. 4.4.1. The intersection point between the two lines matches the nucleus position. PSF profiles are given on the bottom left of each corresponding image. 

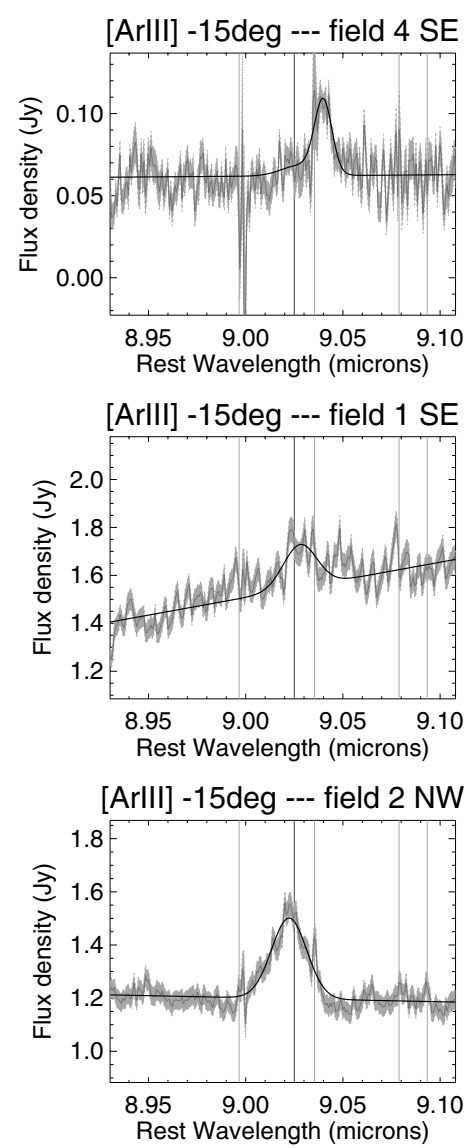
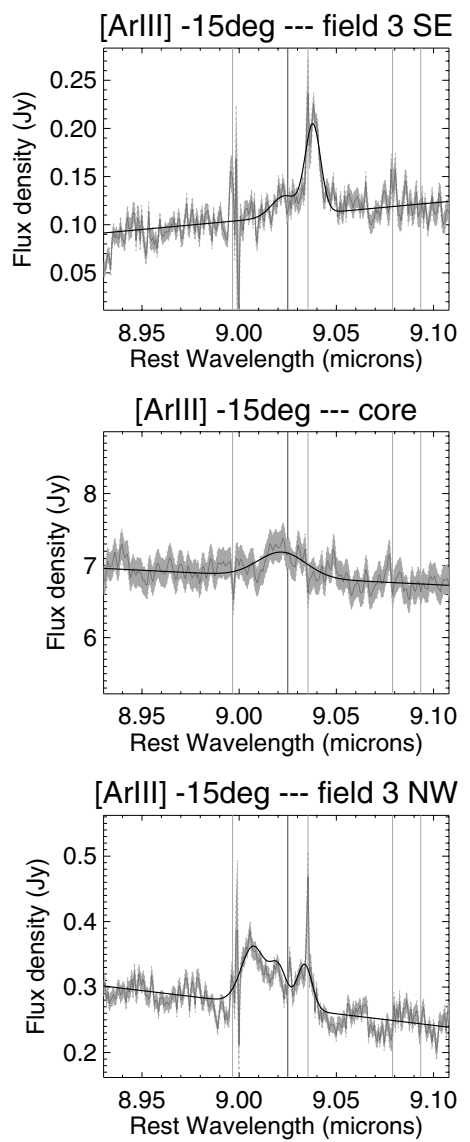
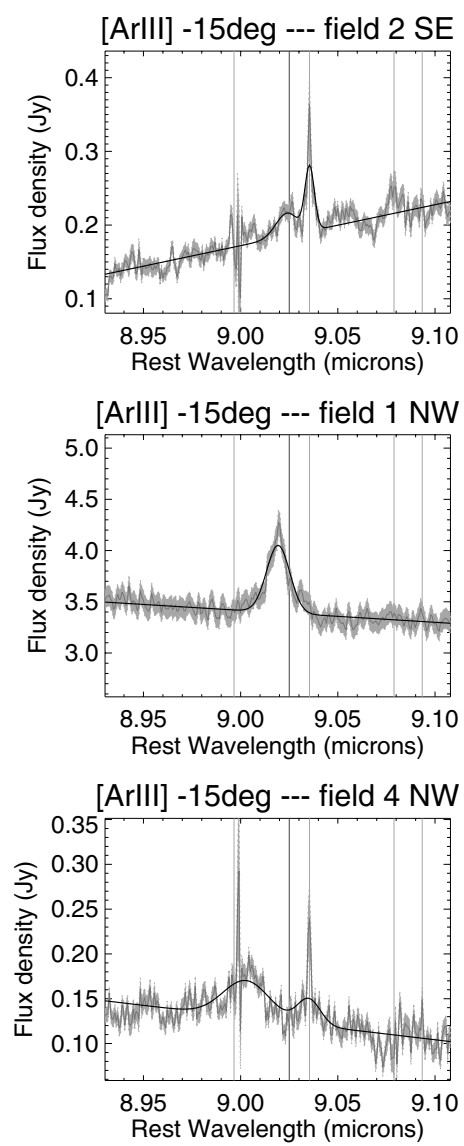

Fig. 2. Spectra in the ArIII $8.99 \mu \mathrm{m}$ filter extracted in 0'.381 steps, slit at PA $=-15^{\circ}$. Reduced spectra and associated error bars are plotted in grey, and fit of the continuum plus Gaussian profiles are in black solid lines. Light and dark grey vertical lines mark the location of sky lines and of the theoretic position of the $[\mathrm{ArIII}]+[\mathrm{MgVII}]$ line at rest $(z=0.003786$, Paturel et al. 2002). (See on-line material for additional spectra from fields $10 \mathrm{SE}$ to $10 \mathrm{NW}$ and those obtained with the slit positioned at $P A=90^{\circ}$.)

Paturel et al. 2002) and corresponding to the three filters and the two slit positions, are presented in Figs. 2 to 4 (see also the on-line material). Even with the resolution power of $R \sim$ 3000 , the [ArIII] line is blended with the [MgVII] line positioned at $\pm 0.004 \mu \mathrm{m}$ from the former (Hayward et al. 1996). This is supported by ISO/SWS spectra $(R \sim 2000)$ which do not individually separate the two lines and by the detection of the [MgVII] line at $5.5 \mu \mathrm{m}$ (Lutz et al. 2000). In what follows, we therefore refer to the line detected in the ArIII filter as $[\mathrm{ArIII}]+[\mathrm{MgVII}]$.

Detected emission lines were fitted with a Gaussian profile. First, the average continuum levels are estimated from fits with a polynomial - one degree is sufficient according to the narrowness of filters. They are then subtracted from spectra and emission lines are fitted with a Gaussian profile, the three free parameters being the position, the width and the height of the line. For each spectrum, error bars on line parameters are estimated from a variation of 1 on the minimal $\chi^{2}$ associated with the fit. Line fluxes (and the associated error bars) are deduced from the emission line parameters (and from the associated error bars). They are then used to compute line ratios for which error bars are estimated from a Monte Carlo calculation. For this, line fluxes are considered as Gaussian random variables, of mean equal to the measured values and the standard deviation equal to the measured error bars. The Monte Carlo processing consists of first generating random intensities for two lines of interest. This is repeated several hundred times. Each time, a corresponding line ratio is estimated via the ratio between the means of generated intensities. The standard deviations of line ratios are considered as the final error bars on ratios.

\section{Results}

\subsection{Description of images}

The deconvolved images presented in Fig. 1 show the spatial distribution of the total flux for each narrow-band filter. Even in filters corresponding to emission line wavelengths, the continuum associated with warm dust is likely to dominate the total flux. We recognize structures similar to the $12.8 \mu \mathrm{m}$ deconvolved VISIR image from Galliano et al. (2005, i.e. knots labeled NE1 to NE4 and SW1, SW2) and the nuclear $S$-shape highlighted by the NIR deconvolved images from Gratadour et al. (2006). The brightest northern and southern knots (at $\sim 0$.' 5 from the core) seem wellresolved in the two NeII filters. In the ArIII filter, the bright nuclear point-source is associated with the emission from the dusty torus resolved by interferometry (Poncelet et al. 2007; Raban et al. 2007, in prep.). We reported on the images the aperture and the PA of the northern ionization cone edges derived by Capetti et al. $\left(1997\right.$, i.e. $55^{\circ}$ and $\left.-35^{\circ}\right)$. The emission is well collimated in the SIV2 and in the two NeII filters, while it is more diffuse in the other filters, especially west of the core in the PAH2 filter. This does not result from PAH emission missing in the few central arcseconds of NGC 1068 (see Siebenmorgen et al. 2004; Mason et al. 2006). Images also highlight the NS asymmetry in the NLR material distribution. We clearly observe the bending of 


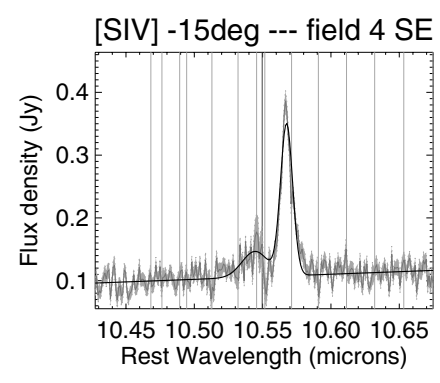

[SIV] -15deg --- field 1 SE
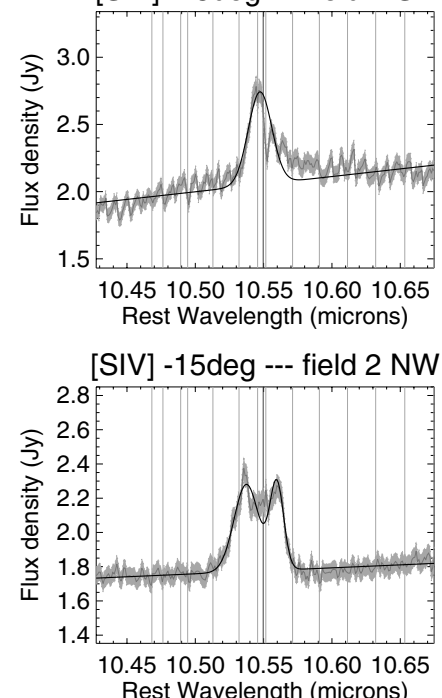

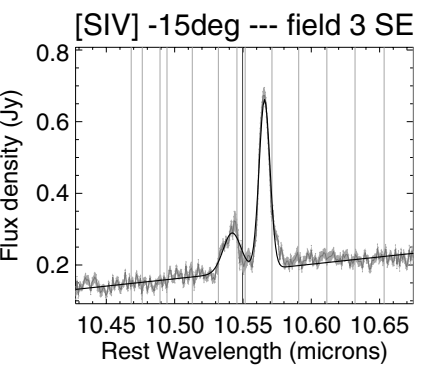

[SIV] -15deg --- core
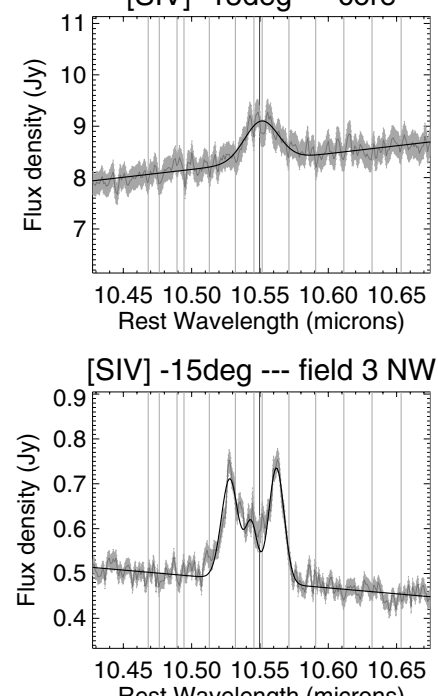

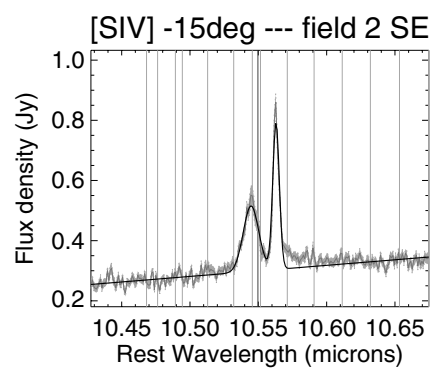

[SIV] -15deg --- field 1 NW
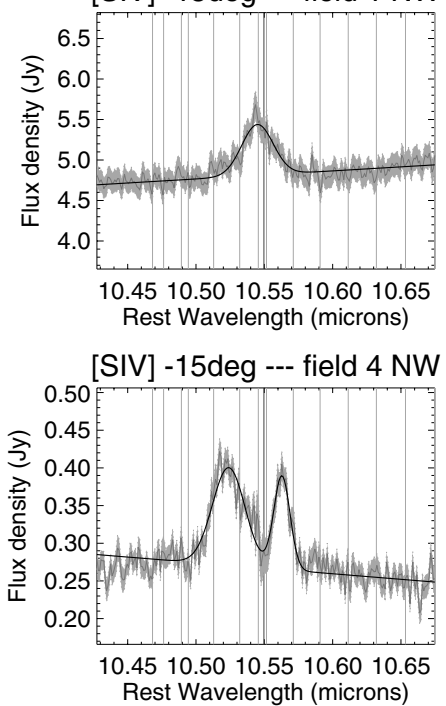

Fig. 3. Spectra in the $S I V 10.51 \mu \mathrm{m}$ filter extracted in 0.381 steps, slit at PA $=-15^{\circ}$. Reduced spectra and associated error bars are plotted in grey, and fit of the continuum plus Gaussian profiles are in black solid lines. Light and dark grey vertical lines mark the location of sky lines and of the theoretic position of the [SIV] line at rest. (All spectra extracted from $10 \mathrm{SE}$ to $10 \mathrm{NW}$ are available on-line.)

structures above the knot NE1, following the same trend as the radio jet. SW of the nucleus, the material also emits along a bar at $\mathrm{PA}=31^{\circ}$ from $0.2 \mathrm{~W}$ to $0.5 \mathrm{E}$, and $0.4 \mathrm{~S}$ to $1^{\prime \prime} .2 \mathrm{~S}$. This may be indirect evidence for the propagation of the counter radio jet (see Sect. 5.3).

\subsection{The nuclear continuum}

Values of the continuum measured from spectroscopy along the two slit positions are listed in Table 2. On the field centered on the core, the average level measured in the ArIII, SIV and NeII filters are $6.8 \pm 0.2 \mathrm{Jy}, 8.3 \pm 0.2 \mathrm{Jy}$ and $12.8 \pm 0.5 \mathrm{Jy}$ for the slit at $-15^{\circ}$ and $7.8 \pm 0.4 \mathrm{Jy}, 10.2 \pm 0.3 \mathrm{Jy}$ and $17.2 \pm 1.2 \mathrm{Jy}$ for the slit at $90^{\circ}$. Error bars were estimated from a simple spectral average of spectra errors bars and not from a quadratic average (the reason for that was to ensure reasonable estimates of error bars associated to parameters from the fit with a black body spectrum). They must therefore be taken as qualitative estimates. The slit at $\mathrm{PA}=-15^{\circ}$ is positioned on the ionization cone where there is more emitting material than along the other direction. Therefore, the differences in flux densities between the two slit positions must originate from a small shift in the positioning of the slits on the core of NGC 1068.

Although it is an oversimplified description, the flux densities measured on the quadrant labeled core in the two slit position were fitted with a black body (BB) spectrum. The silicate absorption feature was not taken into account for the fit. As a consequence, parameters are certainly underestimated, but according to the simplicity of the fit and to the large error bars associated with it (see Fig. 5), the derived size and temperature must be of the right order of magnitude. Indeed, the simple BB fit points towards typical BB temperature and size of $282 \pm 10 \mathrm{~K}$ and $2.8 \pm 0.3$ pc respectively, in agreement with interferometric studies pointing towards a parsec scale torus of temperature $\sim 350 \mathrm{~K}$ (Jaffe et al. 2004; Poncelet et al. 2006). MIR spectroscopy on the core of NGC 1068 was investigated by Mason et al. (2006) with roughly the same spatial resolution $\left(0^{\prime \prime} 4\right)$ and $R \sim 200$. Levels of the continuum at 8.9, 10.5 and $12.8 \mu \mathrm{m}$ are consistent and from these, they derive a typical color temperature of $\sim 330 \mathrm{~K}$. This thermal emission is supposed to originate from the dusty torus of NGC 1068 and they actually succeeded in reproducing the $N$-band spectrum of the core with a model of clumpy torus requiring $90 \%$ of the dusty clouds to lie within $3 \mathrm{pc}$ of the nucleus. All these results are an important clue in favor of clumpy models since they are to date the only description able to explain such low temperatures so close to the central engine.

The continuum flux density decreases rapidly with radius in all directions. The $F W H M$ of the emission peak is $\sim 0$.' 5 for the slit at $\mathrm{PA}=90^{\circ}$ and larger $\left(\sim 00^{\prime} 8\right)$ for the slit at $\mathrm{PA}=-15^{\circ}$. There is actually more emitting gas along this second direction and there is more emission toward $\mathrm{N}$ than $\mathrm{S}$.

\subsection{Spectroscopy along the EW direction}

According to the deconvolved images in the ArIII, SIV and NeII filters presented in Fig. 1 and the results from the speckle processing of $12.8 \mu \mathrm{m}$ VISIR BURST mode images (Poncelet et al. 2007), regions west and east of the core are mainly depleted 


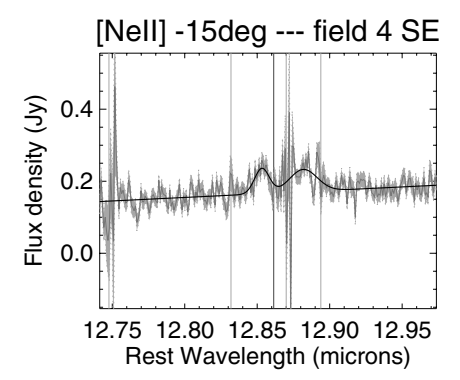

[Nell] -15deg --- field 1 SE
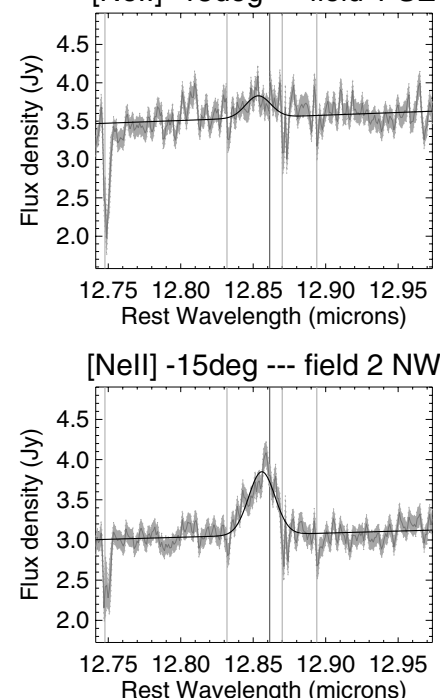
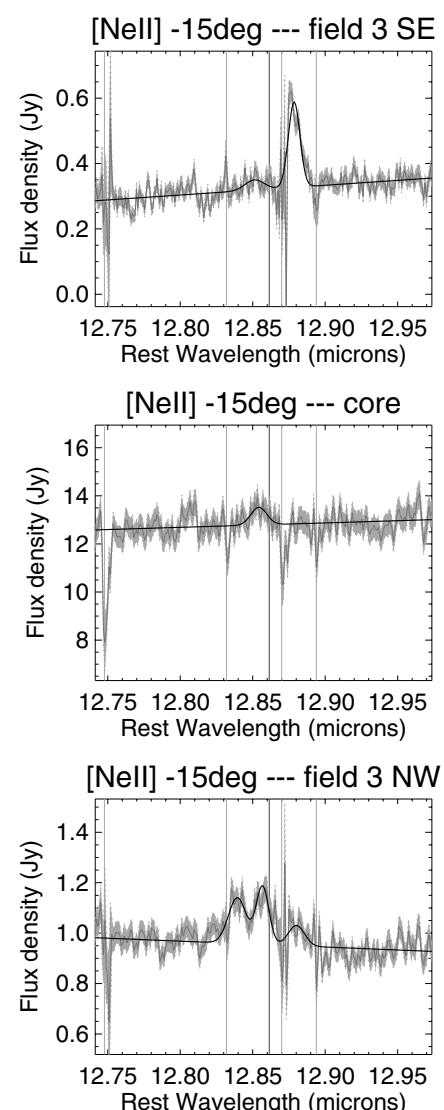

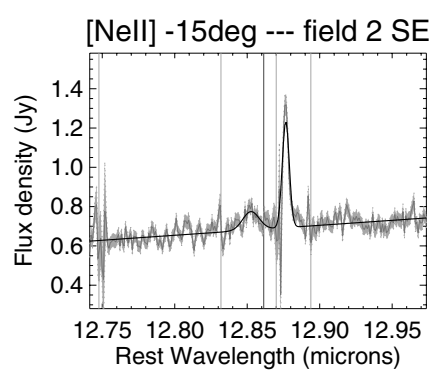

[Nell] -15deg --- field $1 \mathrm{NW}$
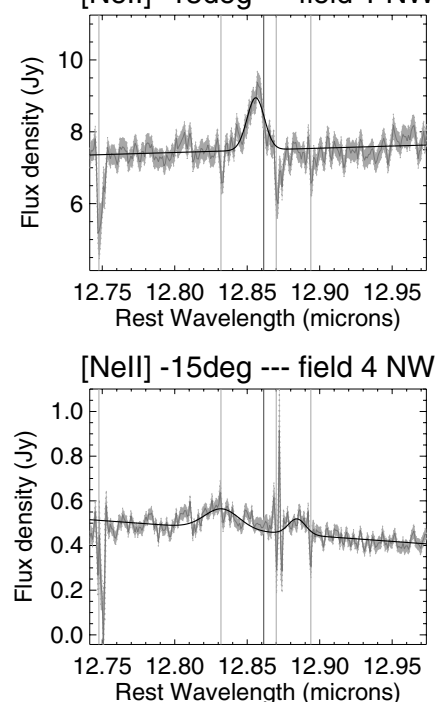

Fig. 4. Spectra in the NeII $12.81 \mu \mathrm{m}$ filter extracted in $0^{\prime} .381$ steps, slit at PA $=-15^{\circ}$. Reduced spectra and associated error bars are plotted in grey, and fit of the continuum plus Gaussian profiles are in black solid lines. Light and dark grey vertical lines mark the location of sky lines and of the theoretic position of the [NeII] line at rest. (All spectra extracted from $10 \mathrm{SE}$ to $10 \mathrm{NW}$ are available on-line.)

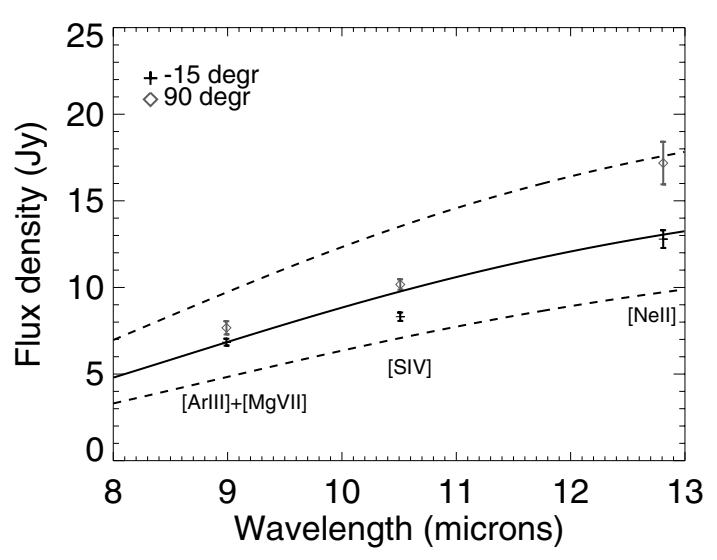

Fig. 5. Continuum on the central $0 . ' 4 \times 0$. '38. Data points and associated error bars correspond to continuum measurements in the three filters. The solid line is the fit by a black body spectrum of temperature $282 \mathrm{~K}$ and a physical size of $\sim 2.8 \mathrm{pc}$. The two dashed lines show the confidence interval in the fit.

of emitting gas or dust. At the time of observations, the slit position at $\mathrm{PA}=90^{\circ}$ was chosen a priori to concentrate the study on the dusty torus, assumed to be the main contributor to the observed flux density along this direction. However since there is less emitting material along this direction, the associated flux densities are weaker and the signal-to-noise ratio is poorer. This especially concerns spectra taken with the ArIII filter. Spectra extracted along this particular slit position are presented in online material.
In the three filters considered, all detected lines appear in emission and none in absorption. The [SIV] line which has the best signal-to-noise ratio is detected from quadrants $2 \mathrm{~W}$ to $4 \mathrm{E}$ (i.e. from $\sim 1^{\prime \prime}$ west to $\sim 1^{\prime \prime} .8$ east of the core). The detection of the $[\mathrm{NeII}]$ line and especially of the $[\mathrm{ArIII}]+[\mathrm{MgVII}]$ lines are less obvious. In each quadrant, a way to disentangle the emission line from noises consists of attempting a fit of these two lines with the same width as the [SIV] line. As shown by Figs. 12 and 14 in the on-line material, the only quadrants where this worked were those labeled $2 W, 1 W$ and $1 E$, although in the quadrant $1 W$, the emission line is very weak and at the limit of detection and in the quadrant $1 E$, the spectrum quality is extremely poor concerning the ArIII filter. This method also worked for the [NeII] line in the quadrants $2 W$ to $4 E$. Average $\chi_{\text {red }}^{2}$ values associated with the fits of spectra (continuum plus emission lines when detected) are $2.14,1.56$ and 1.67 for the three filters respectively.

Radial velocities along the line of sight are derived from the Doppler shift of lines. In the present paper, they are all given relative to the galaxy systemic velocity $\left(1148 \mathrm{~km} \mathrm{~s}^{-1}\right.$; Brinks et al. 1997). Line width is associated with Doppler broadening. Therefore, it results from the sum of two terms: one is thermal agitation and the other is caused by a non-thermal origin which we will call equivalent velocity hereafter, which is either associated with a turbulent velocity or with a velocity dispersion due to the motion of individual gas clouds which have slightly different inclinations along the line of sight. If we assume a maximal gas temperature of $10^{5} \mathrm{~K}$ inside the NLR (see Sect. 4.4.2), line widths are dominated by the non-thermal term of the Doppler broadening. Equivalent velocities measured from line FWHM estimates are reported in Fig. 7, where points of 

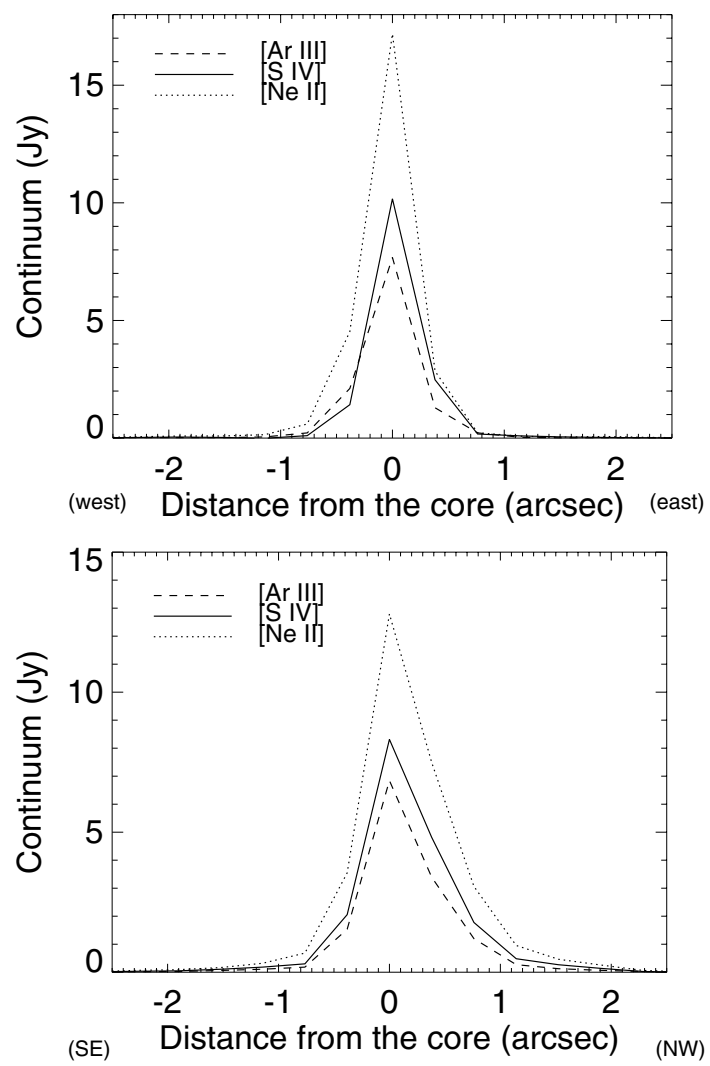

Fig. 6. Evolution of the continuum in directions perpendicular to (slit at $\mathrm{PA}=90^{\circ} ;$ top ) and along the ionization cone (slit at $\mathrm{PA}=-15^{\circ} ;$ bottom). The difference in flux density at peak probably originates from a slight shift in the slit positioning on the core. For the slit at PA $=90^{\circ}$, the left wing in the flux density peak is due to more material in the west of the core (west is toward negative distance), while for the slit at PA $=90^{\circ}$, the more extended right wing in the flux density peak correspond to the northern part of the ionization cone, whose emission suffers less absorption from the host galaxy disk (see Sect. 4.4).

measurement correspond to radial velocities and vertical bars to equivalent velocities. Velocity points associated with the $[\mathrm{ArIII}]+[\mathrm{MgVII}]$ and $[\mathrm{NeII}]$ lines are also reported in in Fig. 7, although they have to be considered with much caution. We observe that the radial velocity field along this slit position has an average value $<100 \mathrm{~km} \mathrm{~s}^{-1}$ and does not depart more than $300 \mathrm{~km} \mathrm{~s}^{-1}$ from the systemic velocity of the galaxy. There is no significant evolution of the velocity field which may be associated with the lateral velocity of infalling materials fuelling the torus. Equivalent velocities (typical value of $250 \mathrm{~km} \mathrm{~s}^{-1}$ ) are on average larger than the radial velocity and are typically larger $\mathrm{W}$ of the core than E (see Fig. 7; up to $600 \mathrm{~km} \mathrm{~s}^{-1}$ ).

\subsection{Spectroscopy across the narrow line region}

The second slit position at $\mathrm{PA}=-15^{\circ}$ was used to scan the NLR. The flux density associated with the continuum is thought to be mostly thermal, i.e. related to the reradiation by dust. Measurements of the flux density of the continuum along this slit position (presented in Table 2) were fitted with a BB spectrum to estimate the typical temperature of the dust inside the NLR - except for the field labeled core for which the temperature has been deduced in Sect. 4.2). The size of the BB was constrained to the size of one quadrant. The typical temperature of dust seen in MIR decreases rapidly from $\sim 300 \mathrm{~K}$ on the core
Table 1. Temperatures across the ionization cone at $-15^{\circ}$, estimated from fits of the continuum flux density points in the ArIII, SIV and NeII filters with a BB spectrum.

\begin{tabular}{ccc}
\hline \hline Field & Distance $\left({ }^{\prime \prime}\right)$ & $T(\mathrm{~K})$ \\
\hline $6 \mathrm{SE}$ & -2.286 & 95 \\
$5 \mathrm{SE}$ & -1.905 & 100 \\
4 SE & -1.524 & 105 \\
3 SE & -1.143 & 110 \\
$2 \mathrm{SE}$ & -0.762 & 120 \\
$1 \mathrm{SE}$ & -0.381 & 145 \\
core & 0 & 282 \\
$1 \mathrm{NW}$ & 0.381 & 160 \\
$2 \mathrm{NW}$ & 0.762 & 145 \\
$3 \mathrm{NW}$ & 1.143 & 125 \\
4 NW & 1.524 & 115 \\
$5 \mathrm{NW}$ & 1.905 & 105 \\
$6 \mathrm{NW}$ & 2.286 & 95 \\
\hline
\end{tabular}

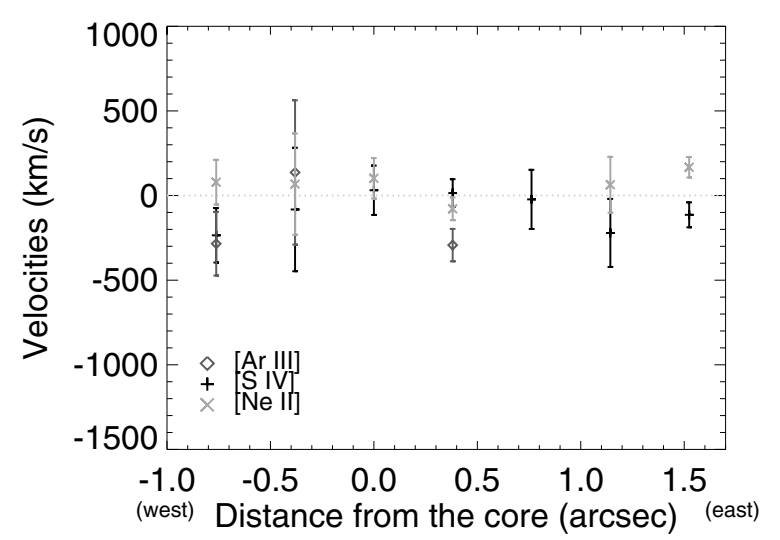

Fig. 7. Radial velocity fields along the slit at PA $=90^{\circ}$ (i.e. positioned EW and centered on the core) derived from positions of the $[\mathrm{ArIII}]+[\mathrm{MgVII}]$, $[\mathrm{SIV}]$ and the $[\mathrm{NeII}]$ lines. Vertical bars are the turbulent velocities of the gas measured from the Doppler broadening of emission lines. Negative distances from the core correspond to the $\mathrm{W}$ and the positive ones to the $\mathrm{E}$.

to $\sim 150 \mathrm{~K}$ at $\sim 0$. $^{\prime} 4 \mathrm{~N}$ and $\mathrm{S}$, then remains of the order of $100 \mathrm{~K}$ beyond.

As for the slit position at $90^{\circ}$, all detected lines are seen here in emission. Among the three filters, the flux of the [SIV] line is the most important. Assuming that in each considered quadrant, the three emission lines originate from the same NLR clouds, the Doppler broadening due to gas motion, i.e. the line width, should be the same for the three lines. The [SIV] lines has therefore been used as a constraint for a better detection of the two other fainter lines and to fit them. This process worked well, except for few quadrants where the [SIV] line width does not reproduce well that of the $[\mathrm{ArIII}]+[\mathrm{MgVII}]$ or the $[\mathrm{NeII}]$ line (see Table 3 for values associated with quadrants $6 \mathrm{SE}, 1 \mathrm{SE}, 2 \mathrm{SE}$, core, $5 \mathrm{NW}$, and $6 \mathrm{NW}$ - in this latter, solely the [SIV] line is detected). Average $\chi_{\text {red }}^{2}$ values associated with the fits of spectra (continuum plus emission lines when detected) are 1.40, 1.21 and 1.33 for the three filters respectively. Line parameters are listed in Table 3 and plotted as a function of the distance from the core in Figs. 8 and 9. The [SIV] line is detected from $\sim 3^{\prime \prime} \mathrm{SE}$ to $\sim 3^{\prime \prime} \mathrm{NW}$ from the core, the $[\mathrm{ArIII}]+[\mathrm{MgVII}]$ line from $\sim 2$ '. $3 \mathrm{SE}$ to $\sim 1^{\prime \prime} .5 \mathrm{NW}$ and the $[\mathrm{NeII}]$ line from $\sim 2$ '. $3 \mathrm{SE}$ to $\sim 2$ '. $7 \mathrm{NW}$, and another time in the field $10 \mathrm{NW}$ corresponding to a distance of 3 ". 8 from the core. This last detection may highlight the location of point-like starburst activities inside the stellar arm joining the nuclear core 

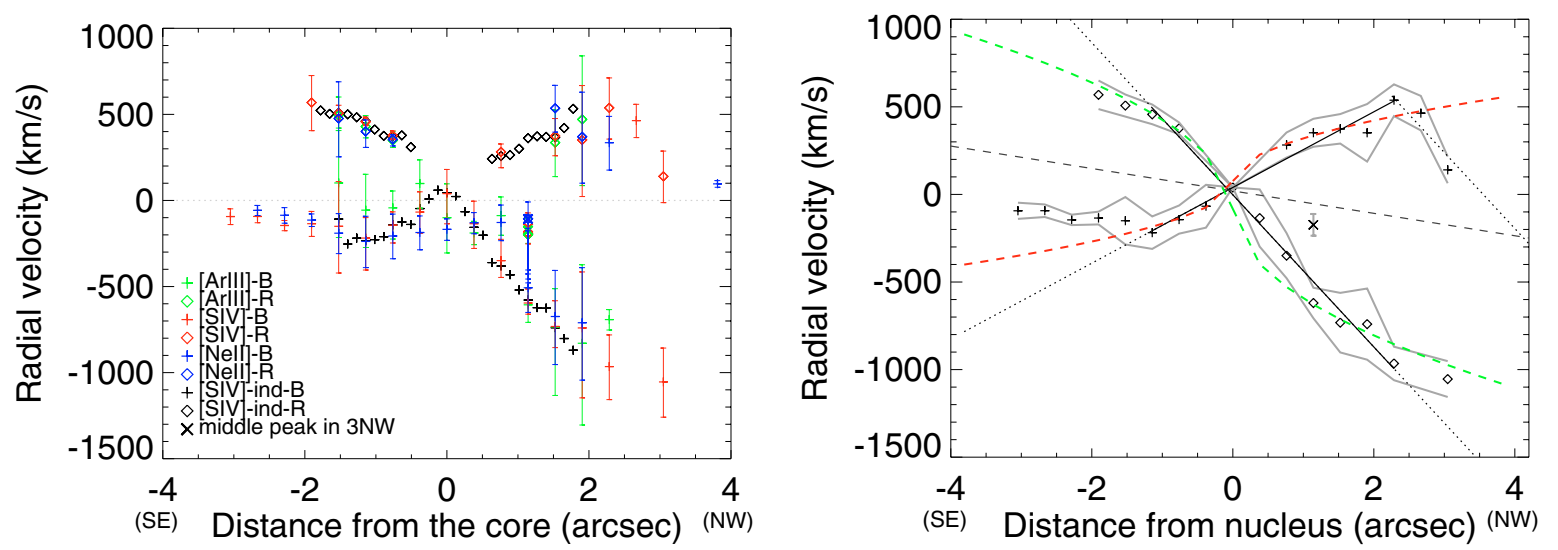

Fig. 8. Radial velocity fields across the ionization bicone of NGC 1068, relative to the systemic velocity of NGC 1068 (1148 km s${ }^{-1}$; Brinks et al. 1997). On the left: velocity measured from the Doppler shift of the twin-peaked lines in the three filters. This proves the hollow nature of the ionization cone. There is a clear center of symmetry which can be associated to the core. Further out toward S, the material decelerates linearly while toward the $\mathrm{N}$ of the core, decelerations are more abrupt. On the right: each edge of the [SIV] velocity profile has been fitted with two velocity laws: $v \propto r$ (dark solid lines) and $v \propto r^{1 / 2}$ (red and green dashed lines). The symmetry between $2^{\prime \prime} \mathrm{NW}$ and 1".2 SE from the core was investigated and highlighted an inclination of the bicone of $\sim 10^{\circ}$ out of the sky plane (see the grey dashed line) and an opening angle of $\sim 82^{\circ}$. Turbulence velocities are also reported on the radial velocity (see the grey solid lines on both sides of the velocity profile).

to the circumnuclear starburst ring at $13^{\prime \prime}$ from the core (see the $\mathrm{CO}(1-0)$ maps from Usero et al. 2004).

Lines are twin-peaked in almost all quadrants except on the three quadrants centered on the core and on the field $3 \mathrm{NW}$ (i.e. 1'. $14 \mathrm{NW}$ of the core) which exhibits a triple-peaked line. This peculiar field will be discussed further in Sect. 5 .

\subsubsection{Velocity profiles}

Velocity fields are presented in Fig. 8. The blueshifted and redshifted lines are well separated, highlighting that lines are emitted by two distinct structures. The evolution is surprisingly linear and mostly centro-symmetric between $1^{\prime \prime}$ SE to $2^{\prime \prime} \mathrm{NW}$ from the core. The blueshifted part toward the NW is accelerated to $\sim 1000 \mathrm{~km} \mathrm{~s}^{-1}$ at $3^{\prime \prime}$. There is a clear deceleration of the material further than $2.3 \mathrm{NW}$ of the nucleus, more spectacularly concerning the outer edge of the ionization cone. The distance where the velocity cancels out toward the $\mathrm{N}$ is $\sim 4$ "' 1 (see Fig. 8)

To constrain the geometry of the structure in which the gas is outflowing, we made use of simple velocity laws to fit velocity points as a function of the radial distance $(r)$ from the core: $v \propto r$ and $v \propto r^{1 / 2}$. The simple " $r$-law" leads to a biconical description with the following parameter estimates: the most distant edge (i.e. responsible for the redshifted velocities) is inclined $-30.4^{\circ}$ out of the sky plane. The closest one is inclined $51.4^{\circ}$, leading to an opening angle of the structure of $\sim 82^{\circ}$, and to an inclination out of the sky plane of $\sim 10^{\circ}$ (toward the observer in the northern part of the nucleus). The " $r^{1 / 2}$-law" leads to a clepsydra geometry with roughly the same estimates. The center of symmetry is slightly shifted by 60 mas SE concerning the " $r$-law" and 130 mas for the " $r^{1 / 2}$-law", i.e. one and a half time the pixel angular size. The center of symmetry is also shifted $25 \mathrm{~km} \mathrm{~s}^{-1}$ toward the positive velocities. According to the spatial resolution and to the error bars associated with velocity profiles, this is consistent with the position of the compact core of NGC 1068. According to fits with the two simple velocity laws, it is clear that what we observe is gas accelerating along a hollow biconical structure. This structure must therefore be associated with the ionization bicone of NGC 1068.
The velocity profiles obtained from ground-based

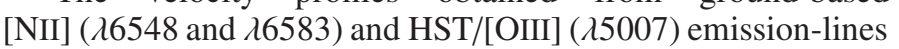
observations by Cecil et al. (1990) and Cecil et al. (2002) are in good agreement with that deduced here. They matched data with a $r^{0.6}$ spatial velocity law and found exactly the same opening angle for the bicone, and an inclination of $5^{\circ}$. These conclusions are supported by the simple biconical outflow models applied to these data by Crenshaw \& Kraemer (2000) and Das et al. (2006). They derive a turn-over radius (i.e. the distance where the gas starts to decelerate) of the bicone of 1".94 and a maximal extension of each side of the bicone along its axis of $5^{\prime \prime} 5$. According to the difference in spatial and velocity resolutions between the two studies, this is consistent with our measurements. Nevertheless, we disagree with the value of $\sim 30^{\circ}$ given by Das et al. (2006) for the bicone orientation in the sky plane since according to its opening-angle, the western edge would be oriented at $\mathrm{PA}=-10^{\circ}$. Here, the double-peaked lines are clearly seen along the slit positioned at $\mathrm{PA}=-15^{\circ}$, meaning that the axis of the ionization bicone is oriented farther toward $\mathrm{W}$, and in the following we adopt an orientation of $10^{\circ}$ highlighted from our narrow-band imaging and in agreement with that deduced by Capetti et al. (1997, see the superposition on the deconvolved images in Fig. 1).

According to Cecil et al. (1990), the radio jet axis is inclined $45^{\circ}$ from the host galaxy for which $\mathrm{PA}=40^{\circ}$ and $i=32^{\circ}$ (Ulvestad \& Wilson 1989). This means that the axis of the ionization cone and of the radio jet basis are aligned. This rules out the possibility that the deceleration observed in the velocity profiles farther than $1^{\prime \prime} \mathrm{SE}$ and $2^{\prime \prime} \mathrm{NW}$ are due to interactions between the outflow and the jet. Additionally, the radio jet is by far more collimated than the ionization cones. Therefore it is unlikely that materials outflowing along the bicone edges are locked to the radio jet by electromagnetic forces and driven by it. The wind is rather thought to be driven by the strong radiative pressure from the central engine (see the scenario proposed by Dopita et al. 2002, for instance).

Considering the inclination of the host galaxy disk, one of the bicone tangents lies on the galactic plane. The peculiar orientation between the structures is further discussed in Sect. 5.2. 

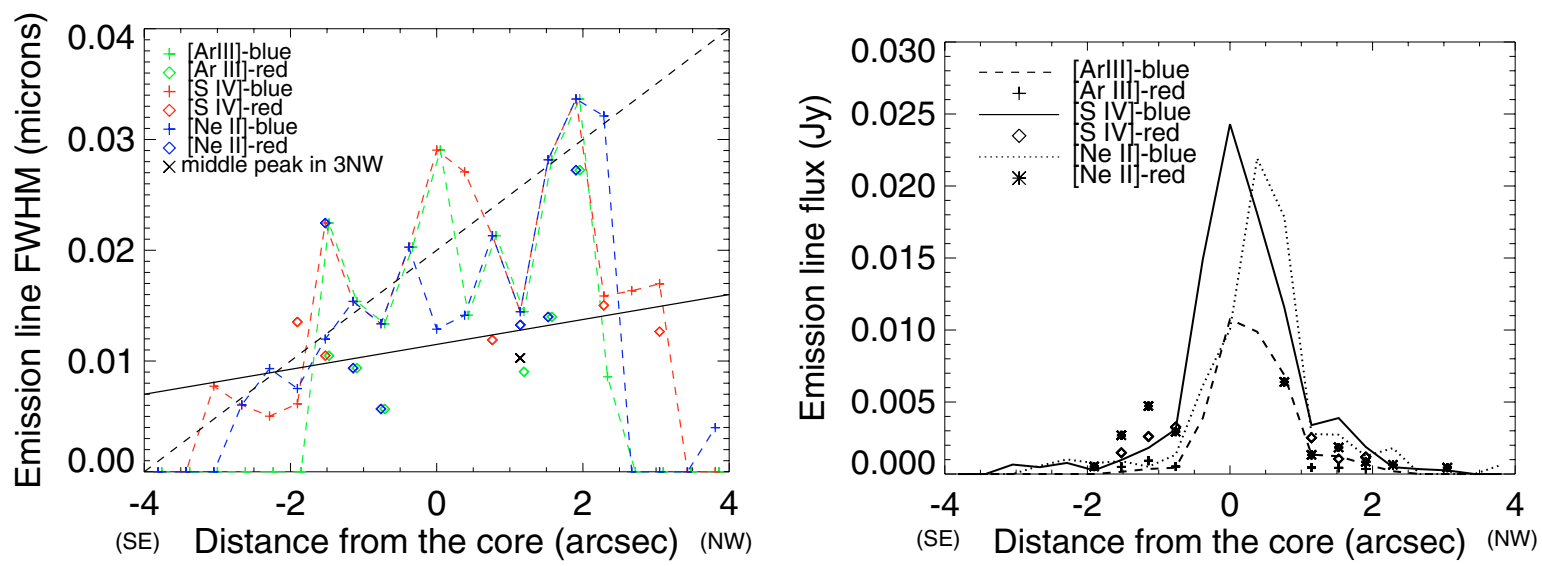

Fig. 9. Spatial evolution of the emission line FWHM (on the left) and fluxes (on the right). FWHM increase from SE to NW of the nucleus. $[\mathrm{ArIII}]+[\mathrm{MgVII}]$ and $[\mathrm{SIV}]$ line fluxes peak at the position of the core, while the [NeII] line flux peaks 0.' $4 \mathrm{NW}$ of the core.

\subsubsection{Evolution of line widths}

The extraction of a typical trend in the line widths evolution is more difficult. However, it is clear that line widths increase from $\mathrm{S}$ to $\mathrm{N}$ of the nucleus, the slope being steeper for the blueshifted line (see the black dotted and black solid lines in Fig. 9).

According to the measured line widths, thermal motions dominate the broadening provided that the gas temperature exceeds $\sim 10^{6} \mathrm{~K}$. We used the maximal temperature of the outflowing wind formula from Krolik \& Begelman (1986) to derive the typical temperature inside the NLR gas. Considering a typical bolometric luminosity of $1.5 \times 10^{11} L_{\odot}$ for the nucleus of NGC 1068 (Bock et al. 2000) and a maximal half-height of the dusty torus of $2 \mathrm{pc}$, the upper value obtained for the temperature close to the core is $\sim 4 \times 10^{6} \mathrm{~K}$. Assuming that the temperature simply decreases as $d^{1 / 2}$ (where $d$ is the distance to the core) inside the NLR, the temperature at 0.5 from the core is an order of magnitude cooler. Moreover, the NLR gas temperatures estimated from fits of X-ray lines range between $3 \times 10^{4} \mathrm{~K}$ and $5 \times 10^{5} \mathrm{~K}$. These arguments show that, apart from the field centered on the central engine of NGC 1068, the Doppler broadening of lines is dominated by the non-thermal term. Equivalent velocities are listed in Table 3 and reported on the right of Fig. 8 (see the solid grey lines). If we interpret the equivalent velocities as a velocity dispersion due to a range of inclination of the outflowing material along the line of sight and following the same " $r$-law", it is possible to derive the bicone edges thickness. It ranges between $\sim 0.2$ and 1 '.'2, corresponding to a divergence angle between $5^{\circ}$ and $10^{\circ}$. Due to projection effects and following this interpretation, in the NW part of the nucleus one would expect the closer edge (i.e. the blueshifted arm) along the line of sight to be thicker than the outer edges (i.e. the redshifted arm), and the contrary in the southern part. According to Fig. 8, we do not observe this trend, the northern and southern blueshifted arms appearing thicker. Furthermore, this supports the NS asymmetry in the distribution of the NLR material.

\subsubsection{Spatial variation of line fluxes}

Measurements of the $[\mathrm{ArIII}]+[\mathrm{MgVII}]$, [SIV] and [NeII] line fluxes are plotted on the right of Fig. 9 and listed in Table 3. There are two noticeable differences in their evolution. First, the level of the $[\mathrm{ArIII}]+[\mathrm{MgVII}]$ line is half that of the two others. Second, while [ArIII] $+[\mathrm{MgVII}]$ and [SIV] line fluxes peak on the core, the $[\mathrm{NeII}]$ line flux peaks $0{ }^{\prime} 4 \mathrm{NW}$ of the nucleus.
This corresponds to the location of the knot labeled NE1 in the $12.8 \mu \mathrm{m}$ image of Galliano et al. (2005). This enhancement of the [NeII] line flux may be related to the jet colliding with this particular NLR cloud. At their peak, the [SIV] and the [NeII] line fluxes are roughly similar.

The evolution of line fluxes follows the same trend as the continuum flux density which decreases more rapidly SE of the core than NW (see Fig. 6 and deconvolved images in the ArIII and NeII filters in Fig. 1). As found by Mason et al. (2006), there is actually more NS asymmetry for the [NeII] line than for the two other lines. This is independent of the absorption by silicates which would be more important for the $[\mathrm{ArIII}]+[\mathrm{MgVII}]$ and [SIV] lines much closer in wavelength to the silicate absorption feature than the [NeII] line. This enhancement is not due to nuclear starburst activities since they do not contribute more than $1 \%$ to the total infrared luminosity from the 0.' 4 centered on the nucleus (Marco \& Brooks 2003). This asymmetry must result in part from the inclination of the host galaxy (32 ${ }^{\circ}$ according to Ulvestad \& Wilson 1989) which causes emissions toward the $\mathrm{S}$ to be partly absorbed. There must also be an asymmetry in the distribution of the NLR material since the redshifted emission line fluxes are fainter $\mathrm{N}$ of the nucleus than $\mathrm{S}$, meaning that there is more material in the front edge of the cone towards $\mathrm{N}$.

\subsection{Line ratios vs. photoionization models}

Thanks to the quality of data, line ratios have been computed for the slit at PA $=-15^{\circ}$ (see Sect. 3). They were compared to standard dust-free models (the gas being depleted or undepleted on heavy elements due to dust formation) and dusty radiation pressure-dominated photoionization models from Groves et al. (2004b), where the presence of dust is completely treated. For each set of models, free parameters investigated are the density at the front edge of the ionized cloud, varying from 100 to $1000 \mathrm{~cm}^{-3}$; the metallicity, from $0.25 Z_{\odot}$ to $4 Z_{\odot}$; the spectral index of the central radiation field, from -1.2 to -2 ; and the ionization parameter at the front edge of the cloud, defined as $U_{0}=S_{\star} / n_{0} c$, where $S_{\star}$ is the entering flux of ionizing photons and $\mathrm{n}_{0}$ the initial density of the cloud. $\log U_{0}$ varies between -4 and 0 .

For the comparison, we plotted three line diagrams: $[\mathrm{ArIII}]+[\mathrm{MgVII}] /[\mathrm{SIV}]$ versus $[\mathrm{SIV}] /[\mathrm{NeII}]$ (referred to as diagram 1), [SIV]/[NeII] versus [ArIII]+[MgVII]/[NeII] (referred to as diagram 2) and $[\mathrm{ArIII}]+[\mathrm{MgVII}] /[\mathrm{NeII}]$ versus 

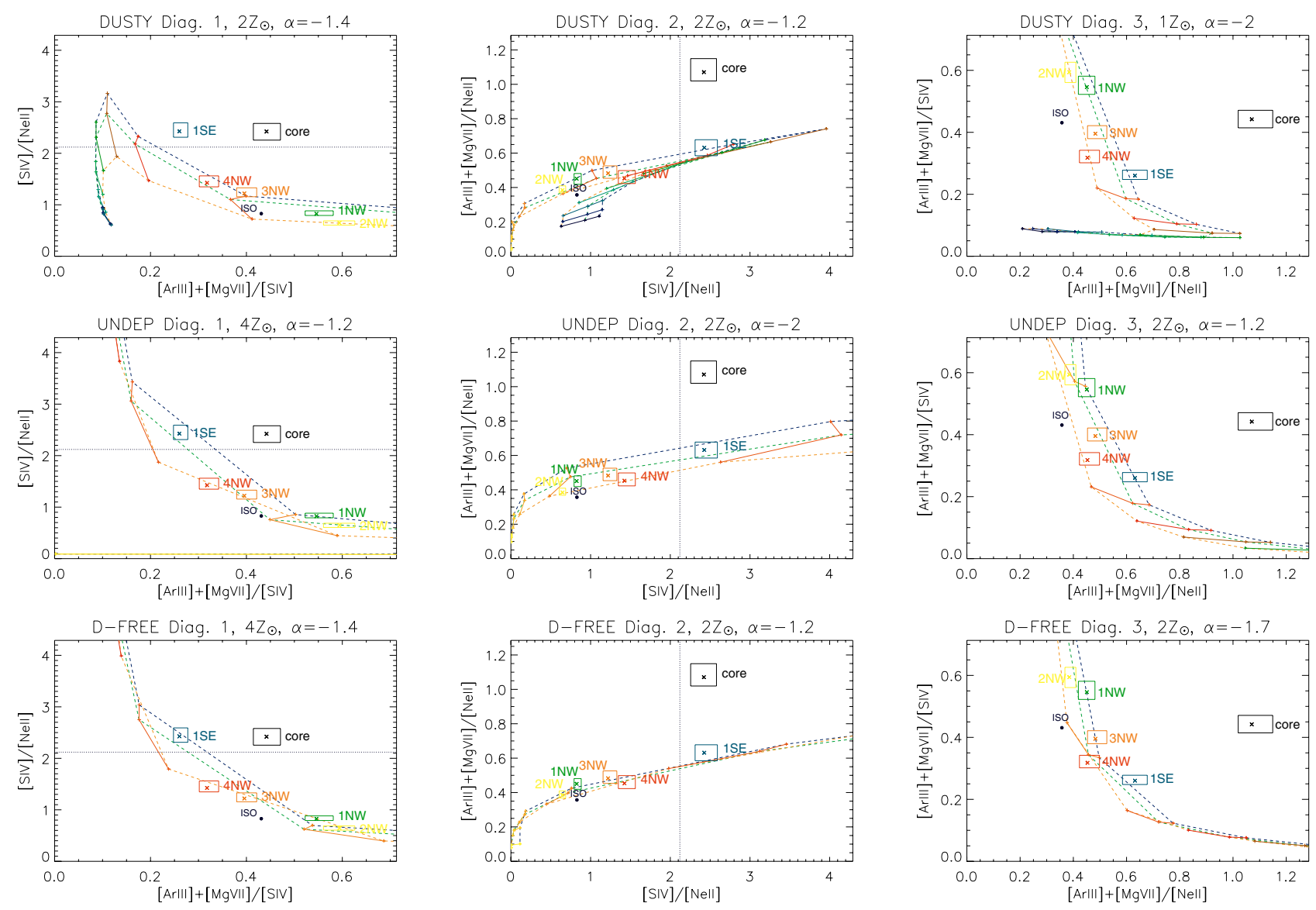

Fig. 10. Comparisons between line ratios derived from VISIR observations and grids of dusty and standard (dust-free and undeleted dust-free) radiation pressure dominated photoionization models from Groves et al. (2004b). The ISO points are displayed by a thick dark blue dot (Lutz et al. 2000). The horizontal and vertical blue dotted lines shown in diagrams 1 and 2 give the value of the [SIV]/[NeII] ratio from TIMMI2 (Siebenmorgen et al. 2004). (Comparisons of all emission line ratios, from field 6 SE to 6 NW including the redshifted lines, with a wider range of photoionization models are available on-line.)

$[\mathrm{ArIII}]+[\mathrm{MgVII}] /[\mathrm{SIV}]$ (referred to as diagram 3). We limited the comparison to lines for having a flux greater than $0.001 \mathrm{Jy}$. The line ratio obtained from the field labeled core (positioned on the central $0 . ' 381 \times 00^{\prime \prime} 4$ ) may be affected by the presence of the dusty torus since it obscures a fraction of the central flux and of lines emitted very close of the central engine. In addition, a fraction of the intensity emitted in the southern part of the ionization bicone must suffer absorption from the host galaxy. Therefore, comparisons in these particular quadrants are to be taken with cautions (this can actually explain the shift between associated data points and model grids; see Figs. 18 to 23 available on-line). As a consequence we limited the comparison to the most reliable estimates of line ratios, i.e. for fields $1 \mathrm{SE}$ to $4 \mathrm{NW}$.

Groves et al. (2004b) did not estimate the emission line intensity for the [MgVII]. As we do not separate the [ArIII] line from the $[\mathrm{MgVII}]$ line, we used the blend as an approximation to plot line ratios. Although according to Groves et al. (2004b), it is dangerous to take a blended line to compute a diagram, this gives a first rough estimate of parameters. Furthermore, diagram 3 is not affected by the use of the blend since the $[\mathrm{ArIII}]+[\mathrm{MgVII}]$ line flux is only used to normalize the flux of the two other lines. The [NeII] line is thought to be enhanced by starburst activity. Here, the line ratio estimates are not affected by this problem since starbursts are detected at $\sim 13^{\prime \prime}$ from the core (Usero et al. 2004), i.e. farther than the detection of lines in the present spectra.
For comparison, we also reported ISO and TIMMI2 line ratios from Lutz et al. (2000) and Siebenmorgen et al. (2004) respectively in Fig. 10. As mentioned previously, there is a strong contribution of starbursts in the ISO aperture which is $14^{\prime \prime} \times 20^{\prime \prime}$. The result is that the ISO point is shifted toward the bottom of Diag. 1 and left of Diag. 3 where the [NeII]line flux is important (see Fig. 10). The line fluxes measured by TIMMI2 correspond to an aperture of $3^{\prime \prime}$, so they do not suffer starburst activities and are directly comparable. However, Siebenmorgen et al. (2004) did not measure the [ArIII]+[MgVII] line flux, so the comparison is only possible with ratios involving the two other lines (see the blue dotted line in Fig. 10 and on-line material). Measurements lie more closely to the core and $1 \mathrm{SE}$ line ratios rather than to that of the northern fields. As the TIMMI2 [SIV]/ [NeII] estimate is an average value over $3^{\prime \prime}$, it must be affected by absorption effects.

In the present case of the NGC 1068's NLR, the three diagrams does not allow us to rule out one of the descriptions (i.e. standard dust-free or dusty photoionization models) proposed by Groves et al. (2004b). Some of the best comparisons are presented in Fig. 10 for fields $1 \mathrm{SE}$ to $4 \mathrm{NW}$ (i.e. the most reliable estimates; see also on-line material for the other line ratios and for a wider range of model parameters). The four northern fields are well fitted by models while the 1 SE lies slightly outward and the field core departs significantly from other line ratio estimates. This shows that the physical conditions inside 

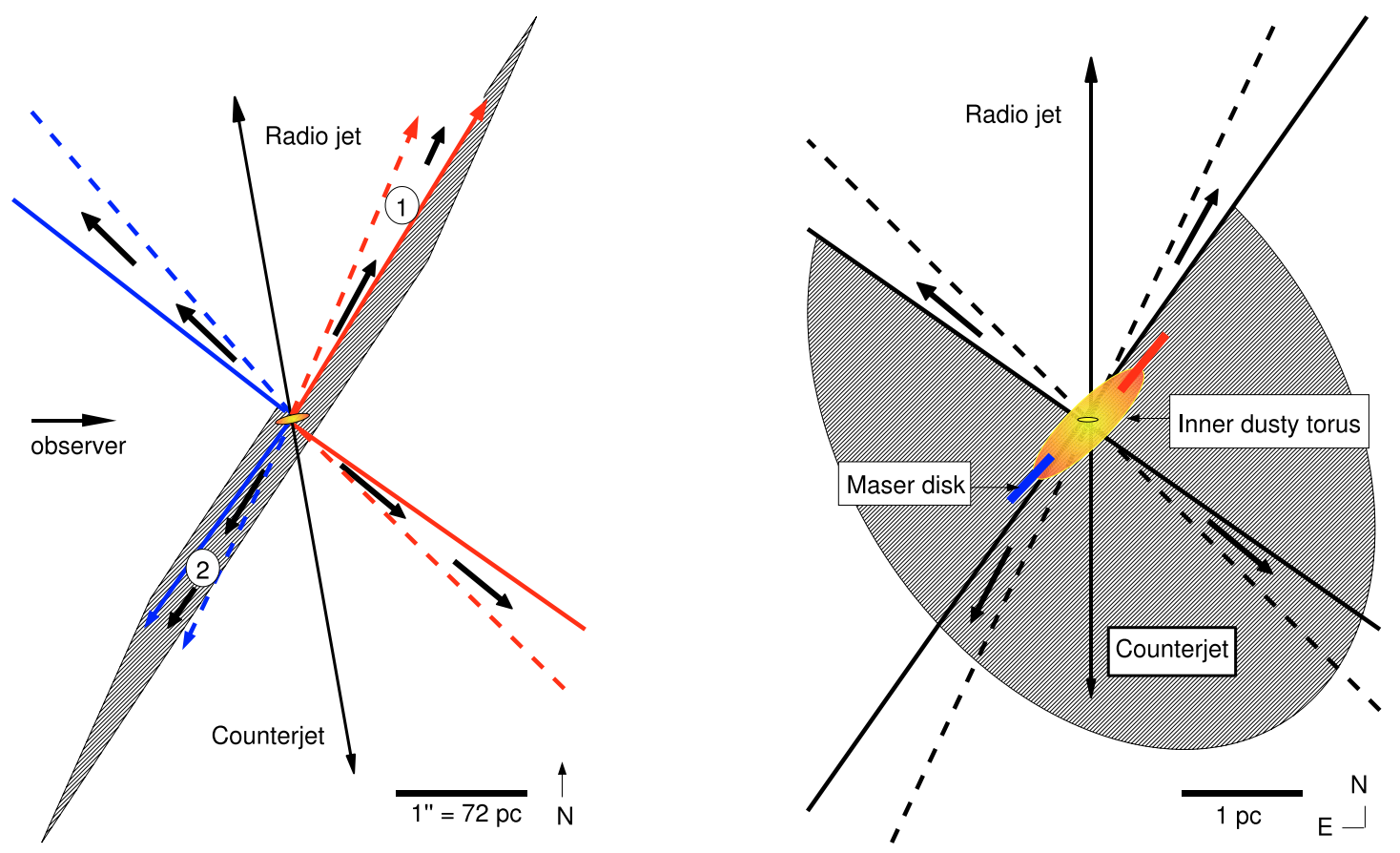

Fig. 11. Different views of the ionization cones. Left: geometry along the line of sight deduced from radial velocity measurements (see Fig. 8 and Sect. 4.4.1). The hatched plane inclined at $\sim-30^{\circ}$ from the observer (placed on the left of the figure) represents the plane of the host galaxy disk. Some interactions between the material outflowing along the bicone walls and the galaxy plane may cause the radial deceleration associated to edges labeled (1) and (2), as illustrated by the arrows (see Sect. 5.2). The radio jet axis is inclined at $45^{\circ}$ from the host galaxy disk, i.e. aligned with that of the ionization cone (Cecil et al. 1990). Right: the ionization bicone as seen in the sky plane, and at a smaller scale. The hatched plane is the host galaxy disk plane. The parsec scale orange component is associated with the inner dusty torus, oriented at $\mathrm{PA}=42^{\circ}$ (as deduced from MIDI observations, Raban et al. 2007). The blue and red lines mark the plane in which the blueshifted and redshifted $\mathrm{H}_{2} 0$ maser spots are distributed. The disk has roughly the same orientation as the inner torus $\left(\mathrm{PA}=40^{\circ}\right.$ according to Gallimore et al. 2001). These latter components are misaligned with the ionization bicone $\left(\mathrm{PA} \sim 10^{\circ}\right.$ ) and the radio jet basis (oriented NS). The initial orientation of the accretion disk is given by the smaller inner disk on the figure.

this peculiar region cannot be entirely described by photoionization models. Based on line ratios of the northern fields, all diagrams provide good constraints on metallicity which must be of the order of $2 Z_{\odot}$ (although values $1 Z_{\odot}$ or $4 Z_{\odot}$ cannot be excluded). This is in good agreement with those derived by Groves et al. (2004c) from the study of a sample of Seyfert 2 galaxies. The density and the spectral index are not constrained here (see Fig. 10).

Concerning the ionization parameter, all northern redshifted line ratios of the three diagrams, for each set of models, are located between $U_{0} \sim 10^{-2.3}$ and $10^{-3.0}$. From MIR spectroscopy with ISO/SWS, Alexander et al. (2000) derived ionization parameters of $10^{-1}$ for the $<1^{\prime \prime}$. 4 inner region, and of $10^{-2}$ up to 4.'5. The discrepancy with the present results may be due to the larger aperture of ISO which collects all the emission from the ionized bicone, while we solely study the particular direction at $\mathrm{PA}=-15^{\circ}$. This is supported by the investigation of HST data by Capetti et al. (1997) who carved the northern part of the NLR into several regions of interest (in particular, see the illustration in Fig. 12 of their paper). The higher ionization region has a typical ionization parameter of $\sim 10^{-2.3}$, and that of the lower, but still highly ionized, is $\sim 10^{-3.2}$. Values $>10^{-2}$ would correspond to an extremely ionized medium such as the coronal line region $\left(U_{0} \sim-1.7\right.$ in the case of NGC 1068, Kraemer \& Crenshaw 2000).

\section{Discussion}

From the study of the emission line profiles, we were able to derive the main characteristics of a hollow biconical structure certainly associated with the ionization bicone (see Sect. 4.4.1). Here we want to discuss the orientation of the structure relatively to the host galaxy disk and to other components in the nucleus, such as the radio jet, the torus, and the maser disk.

\subsection{Implications on the dusty torus and wind geometry}

In the framework of the unified scheme of AGNs, the dusty torus is supposed to define the location and the collimation of the ionization cone. This would imply the axis of the torus to be aligned with that of the ionization cone. This seems not to be the case here according to recent observations of the core of NGC 1068 with MIDI which actually show that the inner torus axis is oriented at $\mathrm{PA}=42^{\circ}$ (Raban et al. 2007, in prep.) rather than the expected $10^{\circ}$ (i.e. the ionization cones PA). Besides, the torus is aligned with the $\mathrm{H}_{2} 0$ masers disk $\left(\mathrm{PA}=40^{\circ}\right.$, Gallimore et al. 2001). Such a layout between these latter structures was expected according to the masers study of Maloney (2002) who claims that the dusty torus is a favorable environment for maser emissions.

Here, we suggest that the misalignment between the ionization cone axis and that of the torus results from a warping of the accretion disk. This hypothesis is based on the BardeenPetterson radius $\left(r_{\mathrm{BP}}\right)$ defined as the distance over which the accretion disk angular momentum is determined by that of the central black hole $(\mathrm{BH})$. Such a warp of the accretion disk is thought to be a common situation in AGNs and this gave rise to a number of recent theoretical studies (see Nayakshin 2005; Lodato \& Pringle 2006, for instance). It has been investigated 
in the particular case of NGC 1068 by Caproni et al. (2006) to explain the misalignment between the maser disk and the radio jet. From the modeling of various observations on the nucleus of NGC 1068, the estimate of $r_{\mathrm{BP}}$ they derived ranges between $10^{-4} \mathrm{pc}$ and $10^{-5} \mathrm{pc}$ (i.e. 40 to 400 Schwarzschild radius $r_{\mathrm{s}}$ ). The angular momentum beyond this radius is that of the infalling accreting material - i.e. that of the dusty torus $\left(42^{\circ}\right)-$ and this change in direction may be the signature of the warping of the disk.

According to wind models of Elvis (2000) and Nicastro (2000), the outflowing material forming the walls of the ionization cone is uplifted from the inner parts of the accretion disk, typically at radius $\lesssim 10^{3} r_{\mathrm{s}}$. Therefore, the outflowing material originates from the inner parts of the warped accretion disk, at smaller distances than the resolved parsec scale torus. The "wind" carries off most of the black hole angular momentum explaining the good alignment of the ionization cone with the radio jet basis which propagates along the $\mathrm{BH}$ spin (oriented mostly NS and inclined at $\sim 10^{\circ}$, Gallimore et al. 2004).

The layout of structures inside the nucleus of Circinus supports this interpretation. The distribution of $\mathrm{H}_{2} \mathrm{O}$ masers actually highlights a warping between the inner disk (on scales $\lesssim 0.1 \mathrm{pc}$ ) - whose axis is more or less aligned with that of the radio lobes and that of the ionization cones - and the outer disk (on scales of $0.4 \mathrm{pc}$ ) which is oriented at $27^{\circ}$ from the inner one (Greenhill et al. 2003).

\subsection{Interactions of the outflowing gas with the host galaxy}

It is very striking that one of the bicone tangents is contained in the host galaxy plane (inclined at $32^{\circ}$, Ulvestad \& Wilson 1989). The velocity profile associated with this tangent (corresponding to the farther edge toward $\mathrm{N}$ and the closer edge toward S) is well-fitted either by the " $r$-law" or the " $r^{1 / 2}$-law" up to 1 .' $5 \mathrm{SE}$ and 2.'3 NW, then significantly departs. Concerning the symmetrical tangent, it is well-fitted up to ionization bicone apexes. One possibility to explain this profile is that the deceleration is due to collisions between the accelerating NLR clouds and the host galaxy disk material. This was proposed by Crenshaw \& Kraemer (2000) according to velocity fields traced by [OIII] lines. The galactic ISM must be anisotropically distributed since the slope of the deceleration profile and the velocity turn-over are slightly different between $\mathrm{N}$ and $\mathrm{S}$. In addition, the slopes of the acceleration and deceleration toward the $\mathrm{N}$ have roughly the same absolute value, possibly highlighting a reflection of the material inward due to the collision with the host galaxy disk.

An interesting feature is also the triple-peak exhibited by the $[\mathrm{ArIII}]+[\mathrm{MgVII}]$, [SIV] and [NeII] lines a in quadrant $3 \mathrm{NW}$ ( $\sim 1$ '. 14 from the core). This is actually the location of an emissive MIR cloud, especially discernible in filters of wavelength longward to $11 \mu \mathrm{m}$ in the deconvolved images in Fig. 1 (see in particular those corresponding to the $\mathrm{PAH} 2, \mathrm{NeII}$ or NeII2 filter). This quadrant actually approximately corresponds to the location of cloud E in the HST/[OIII] image of Macchetto et al. (1994). The lines middle-peak may be enhanced by a collision between the outflowing gas and a dense cloud of the ISM. There is no obvious radio counterpart to this IR/optical cloud (see radio emissions reported by Gallimore et al. 2004). According to Cecil et al. (2002), the mass of cloud HST-E is $\sim 97 \times 10^{2} M_{\odot} / n_{e, 4}$ (where $n_{e, 4}$ is the density in units of $10^{4} \mathrm{~cm}^{-3}$ ), almost twice as massive as the cloud HST-C for instance. Such a collision between the outflow and this cloud would imply different velocity regimes depending on the initial velocity of the ambient medium. Average velocities of $-535 \mathrm{~km} \mathrm{~s}^{-1}$ and $345 \mathrm{~km} \mathrm{~s}^{-1}$ respectively associated with the blueshifted and the most redshifted peaks may correspond to the outflow velocities, while the velocity of $\sim-100 \mathrm{~km} \mathrm{~s}^{-1}$ of the middle-peak may be related to the collision.

\subsection{Interactions between the radio-structures and the NLR gas}

Beyond 4" from the core, HST images show a sharp and well defined boundary in the ionization structure of the NLR (Capetti et al. 1997). This corresponds exactly to the transition between the jet-like and the lobe-like structure of the radio emission (Wilson \& Ulvestad 1987) which is certainly the consequence of bow shocks with the surrounding ISM. Capetti et al. (1997) found the ionization degree to be 3 to 4 times higher at this peculiar location than in the low ionization region of the NLR. Considering that it roughly corresponds to the extreme end of the ionization cone, the velocity turnover at $\sim 2.5^{\prime \prime}$ may be the consequence of two linked effects which causes the material deceleration: the back-flow implied by the boundary shock at $4^{\prime \prime}$ and the interactions of the outflow with the material distributed in the host galaxy disk (essentially toward 2'.3 NW from the core; see Sect. 5.2).

In the inner $4^{\prime \prime}$ of the NLR, there are some local interactions between the radio jet and NLR materials. The peak of the [NeII] line flux at 0.'4 toward NW of the nucleus (fields labeled $1 \mathrm{NW}$ ) may be the consequence of a collision between the radio jet and the NLR cloud labeled NE1 in the MIR and C in the optical (Galliano et al. 2005; Macchetto et al. 1994, respectively).

On the southern part of the nucleus, deconvolved MIR images highlight a peculiar tongue extending up to 1 .' $3 \mathrm{SW}$ (see Fig. 1). It is initially oriented at PA $\sim-15^{\circ}$ up to 0 .' $^{\prime} \mathrm{SE}$ from the core, then bends at $\mathrm{PA} \sim 31^{\circ}$. This is the same behavior as the one exhibited by the radio jet and the UV, optical and IR emissions $\mathrm{N}$ of the nucleus. This argues in favor of a jet-ISM collision at $\$ 0$ '. $4 \mathrm{SE}$ - equivalent to that occurring $\mathrm{N}$ - and the MIR tongue would then be the signature of the propagation of the counterjet interacting with the NLR material. Gallimore et al. (2004) interpreted the component labeled S2 in the $1.4 \mathrm{GHz}$ and $5 \mathrm{GHz}$ radio maps, distant by $\sim 150$ mas from the core, as the signature of a collision between a cloud in the southern part of the ionization bicone and the radio counter jet. The tongue basis has a NIR counterpart highlighted by deconvolution (Gratadour et al. 2006). This latter, located at a distance $\gtrsim 300$ mas $S$ of the core, appears in the deconvolved images in Fig. 1. All these elements support that the basis of the radio counterjet propagates at $\mathrm{PA}=-4^{\circ}$ and twists to $\mathrm{PA}=31^{\circ}$ beyond $0.7 \mathrm{~S}$ from the core .

\section{Summary}

We present medium spectral resolution spectroscopic data obtained with VISIR on the nucleus of NGC 1068. Spectra have been obtained in the ArIII $8.99 \mu \mathrm{m}, S I V \quad 10.51 \mu \mathrm{m}$ and NeII $12.81 \mu \mathrm{m}$ filters along two position slit of interest: $\mathrm{PA}=-15^{\circ}$ and $\mathrm{PA}=90^{\circ}$, to map the spatial evolution of the $[\mathrm{ArIII}]+[\mathrm{MgVII}],[\mathrm{SIV}]$ and $[\mathrm{NeII}]$ lines across the NLR and in a perpendicular direction.

The double-peaked nature of lines with the slit positioned at $\mathrm{PA}=-15^{\circ}$ is evidence for the hollow nature of the observed structure of gas. Fits of the measured velocity profiles (with values of a few hundred $\mathrm{km} \mathrm{s}^{-1}$ up to $1000 \mathrm{~km} \mathrm{~s}^{-1}$ at $3^{\prime \prime} \mathrm{N}$ ) strengthen the biconical geometry and prove that the material 
is outflowing along the edges of the ionization cones. This is inclined at $\sim 10^{\circ}$ toward the $\mathrm{N}$ in direction of the observer and its half-opening angle is $\sim 41^{\circ}$. It is the first time that MIR spectroscopy probed such a structure, in good agreement with previous results of optical spectroscopy. Furthermore, the outflow divergence angle constrained from turbulent velocity estimates ranges between $5^{\circ}$ and $10^{\circ}$. Such an outflow is not observed along PA $=90^{\circ}$ and the weak velocities measured along this direction could be related to infalling material which fuel the obscuring torus.

Line ratio diagrams plotted from the MIR [ArIII $]+[\mathrm{MgVII}]$, [SIV] and [NeII] line fluxe measurements across the NLR of NGC 1068 do not provide diagnostics to discriminate between the standard dust-free and dusty photoionisation models. However, fits of line ratios associated with the northern ionization cone give information on some physical parameters on the emitting gas at $\mathrm{PA}=-15^{\circ}$ : the abundance is close to the solar value and the ionization parameter is of the order of $10^{-2.6}$, corresponding to the highly ionized region of the NLR.

One of the bicone tangents lies within the host galaxy plane. Some local interactions between the outflow and the anisotropic galactic ISM may cause the observed deceleration of material along this tangent.

It appears that the ionization bicone axis is misaligned with that of the inner silicate torus highlighted by interferometry. The former is aligned with the radio jet basis, showing that the outflow forming the bicone walls must originate from small scales in the accretion disk, where the $\mathrm{BH}$ angular momentum dominates. A coherent global picture of NGC 1068 then emerges while considering the layout between the various components inside the nucleus.

Acknowledgements. Eric Pantin for providing the pipeline for the reduction of VISIR data, Cyril Tasse for his great help in IDL. Klaus Meisenheimer and Konrad Tristram for the data acquisition at Paranal, and Walter Jaffe the principal investigator of a MIDI + VISIR observing proposal. Catherine Boisson for beneficial comments on the data reduction and results, Konrad Tristam for his careful and really helpful reading of the article, Brent Groves for advice about the comparison with photoionization models, David Raban for discussion about the obscuring torus, and the referee for improving the article by questions and comments. This work also received the support of PHASE, the high angular resolution partnership between ONERA, Observatoire de Paris, CNRS and University Denis Diderot Paris 7.

\section{References}

Alexander, T., Lutz, D., Sturm, E., et al. 2000, ApJ, 536, 710

Antonucci, R. R. J., \& Miller, J. S. 1985, ApJ, 297, 621
Bergeron, J., Petitjean, P., \& Durret, F. 1989, A\&A, 213, 61

Bock, J. J., Neugebauer, G., Matthews, K., et al. 2000, AJ, 120, 2904

Brinks, E., Skillman, E. D., Terlevich, R. J., \& Terlevich, E. 1997, Ap\&SS, 248, 23

Capetti, A., Macchetto, F. D., \& Lattanzi, M. G. 1997, ApJ, 476, L67

Caproni, A., Abraham, Z., \& Mosquera Cuesta, H. J. 2006, ApJ, 638, 120

Cecil, G., Bland, J., \& Tully, R. B. 1990, ApJ, 355, 70

Cecil, G., Dopita, M. A., Groves, B., et al. 2002, ApJ, 568, 627

Cohen, M., Walker, R. G., Carter, B., et al. 1999, AJ, 117, 1864

Crenshaw, D. M., \& Kraemer, S. B. 2000, ApJ, 532, L101

Das, V., Crenshaw, D. M., Kraemer, S. B., \& Deo, R. P. 2006, AJ, 132, 620

Dopita, M. A., Groves, B. A., Sutherland, R. S., Binette, L., \& Cecil, G. 2002, ApJ, 572, 753

Elvis, M. 2000, ApJ, 545, 63

Galliano, E., \& Alloin, D. 2002, A\&A, 393, 43

Galliano, E., Pantin, E., Alloin, D., \& Lagage, P. O. 2005, MNRAS, 363, L1

Gallimore, J. F., Henkel, C., Baum, S. A., et al. 2001, ApJ, 556, 694

Gallimore, J. F., Baum, S. A., \& O’Dea, C. P. 2004, ApJ, 613, 794

Gratadour, D., Rouan, D., Mugnier, L. M., et al. 2006, A\&A, 446, 813

Greenhill, L. J., Booth, R. S., Ellingsen, S. P., et al. 2003, ApJ, 590, 162

Greenstein, J. L., \& Schmidt, M. 1964, ApJ, 140, 1

Groves, B. A., Cecil, G., Ferruit, P., \& Dopita, M. A. 2004a, ApJ, 611, 786

Groves, B. A., Dopita, M. A., \& Sutherland, R. S. 2004b, ApJS, 153, 9

Groves, B. A., Dopita, M. A., \& Sutherland, R. S. 2004c, ApJS, 153, 75

Hayward, T. L., Saizar, P., Gehrz, R. D., et al. 1996, ApJ, 469, 854

Jaffe, W., Meisenheimer, K., Röttgering, H. J. A., et al. 2004, Nature, 429, 47

Kraemer, S. B., \& Crenshaw, D. M. 2000, ApJ, 532, 256

Krolik, J. H., \& Begelman, M. C. 1986, ApJ, 308, L55

Lodato, G., \& Pringle, J. E. 2006, MNRAS, 368, 1196

Lutz, D., Sturm, E., Genzel, R., et al. 2000, ApJ, 536, 697

Macchetto, F., Capetti, A., Sparks, W. B., Axon, D. J., \& Boksenberg, A. 1994, ApJ, 435, L15

Maloney, P. R. 2002, Publ. Astron. Soc. Australia, 19, 401

Marco, O., \& Brooks, K. J. 2003, A\&A, 398, 101

Mason, R. E., Geballe, T. R., Packham, C., et al. 2006, ApJ, 640, 612

Nayakshin, S. 2005, MNRAS, 359, 545

Nicastro, F. 2000, ApJ, 530, L65

Oliva, E., Marconi, A., Maiolino, R., et al. 2001, A\&A, 369, L5

Pantin, E. 2007, in preparation

Paturel, G., Dubois, P., Petit, C., \& Woelfel, F. 2002, LEDA, 0

Poncelet, A., Doucet, C., Perrin, G., Sol, H., \& Lagage, P. O. 2007, A\&A, 472, 823

Poncelet, A., Perrin, G., \& Sol, H. 2006, A\&A, 450, 483

Raban, D., Jaffe, W., Röttgering, H. J. A., Meisenheimer, K., \& Tristram, K. 2007, in preparation

Rhee, J. H., \& Larkin, J. E. 2006, ApJ, 640, 625

Siebenmorgen, R., Krügel, E., \& Spoon, H. W. W. 2004, A\&A, 414, 123

Ulvestad, J. S., \& Wilson, A. S. 1989, ApJ, 343, 659

Usero, A., García-Burillo, S., Fuente, A., Martín-Pintado, J., \& RodríguezFernández, N. J. 2004, A\&A, 419, 897

van der Kruit, P. C., Oort, J. H., \& Mathewson, D. S. 1972, A\&A, 21, 169

Varosi, F., \& Landsman, W. B. 1993, in Astronomical Data Analysis Software and Systems II, ed. R. J. Hanisch, R. J. V. Brissenden, \& J. Barnes, ASP Conf. Ser., 52, 515

Wilson, A. S., \& Ulvestad, J. S. 1987, ApJ, 319, 105 
A. Poncelet et al.: Dynamics of the ionization bicone of NGC 1068 probed in mid-infrared with VISIR, Online Material $p 1$

Table 2. Average flux density of the continuum (in Jy) in the three narrow-band filters and on each extracted quadrants along the two slit positions $\left(\mathrm{PA}=90^{\circ}\right.$ and $\mathrm{PA}=-15^{\circ}$, negative distances refer to $\mathrm{W}$ and to $\mathrm{SE}$ of the core respectively; significant numbers are given according to the associated error bars on measurements).

\begin{tabular}{c|ccc|ccc}
\hline \hline $\begin{array}{c}\text { Distance from } \\
\text { the core }\left({ }^{\prime \prime}\right)\end{array}$ & {$[\mathrm{ArIII}]+[\mathrm{MgVII}]$} & $\begin{array}{c}90^{\circ} \\
{[\mathrm{SIV}]}\end{array}$ & {$[\mathrm{NeII}]$} & {$[\mathrm{ArIII}]+[\mathrm{MgVII}]$} & $\begin{array}{c}-15^{\circ} \\
{[\mathrm{SIV}]}\end{array}$ & {$[\mathrm{NeII}]$} \\
\hline-3.810 & 0.001 & 0.001 & 0.002 & 0.005 & 0.002 & 0.001 \\
-3.429 & 0.005 & 0.002 & 0.01 & 0.004 & 0.005 & 0.01 \\
-3.048 & 0.007 & 0.005 & 0.01 & 0.010 & 0.011 & 0.02 \\
-2.667 & 0.01 & 0.011 & 0.02 & 0.011 & 0.014 & 0.02 \\
-2.286 & 0.02 & 0.026 & 0.06 & 0.018 & 0.03 & 0.06 \\
-1.905 & 0.02 & 0.045 & 0.08 & 0.025 & 0.05 & 0.09 \\
-1.524 & 0.02 & 0.027 & 0.09 & 0.06 & 0.11 & 0.17 \\
-1.143 & 0.05 & 0.009 & 0.14 & 0.10 & 0.18 & 0.32 \\
-0.762 & 0.22 & 0.10 & 0.60 & 0.18 & 0.30 & 0.69 \\
-0.381 & 2.1 & 1.42 & 4.5 & 1.53 & 2.06 & 3.5 \\
0. & 7.7 & 10.2 & 17.1 & 6.8 & 8.3 & 12.8 \\
0.381 & 1.29 & 2.48 & 2.8 & 3.39 & 4.8 & 7.5 \\
0.762 & 0.22 & 0.17 & 0.23 & 1.20 & 1.78 & 3.1 \\
1.143 & 0.03 & 0.09 & 0.08 & 0.27 & 0.48 & 0.95 \\
1.524 & 0.01 & 0.05 & 0.04 & 0.12 & 0.27 & 0.46 \\
1.905 & 0.019 & 0.03 & 0.07 & 0.07 & 0.14 & 0.26 \\
2.286 & 0.015 & 0.014 & 0.03 & 0.02 & 0.02 & 0.06 \\
2.667 & 0.008 & 0.003 & 0.01 & 0.01 & 0.005 & 0.02 \\
3.048 & 0.0009 & 0.002 & 0.003 & 0.007 & 0.002 & 0.010 \\
3.429 & 0.003 & 0.004 & 0.001 & 0.001 & 0.005 & 0.006 \\
3.810 & 0.002 & 0.003 & 0.001 & 0.001 & 0.001 & 0.021 \\
\hline
\end{tabular}


A. Poncelet et al.: Dynamics of the ionization bicone of NGC 1068 probed in mid-infrared with VISIR, Online Material $p 2$

Table 3. Emission line properties as a function of the distance to the core. The table lists measurements of the radial velocity, the $F W H M$, the turbulent velocity and the line flux (significant numbers are given according to the associated error bars on measurements). As far as possible, the $F W H M$ of the [S IV] line was used as a constraint for the fit of the other lines (see Sect. 4.4); when the FWHM is not the same for the three lines, it shows where the [S IV] line width was not representative of that of the other lines. Turbulent velocities inside the gas are calculated from the measurements of the line FWHM.

\begin{tabular}{|c|c|c|c|c|c|c|c|c|c|c|c|c|}
\hline & \multicolumn{3}{|c|}{ Radial velocity } & \multicolumn{3}{|c|}{$\overline{F W H M}$} & \multicolumn{3}{|c|}{ Turbulent velocity } & \multicolumn{3}{|c|}{ Flux } \\
\hline Distance to core & {$[\mathrm{ArIII}]+[\mathrm{MgVII}]$} & {$[$ SIV $]$} & {$[\mathrm{NeII}]$} & {$[$ ArIII $]+[\mathrm{MgVII}]$} & ] [SIV] & {$[\mathrm{NeII}]$} & {$[$ ArIII $]+[\mathrm{MgVII}]$} & [SIV] & {$[\mathrm{NeII}]$} & {$[$ ArIII $]+[\mathrm{MgVII}]$} & {$[$ SIV] } & {$[\mathrm{NeII}]$} \\
\hline (") & $\mathrm{km} \mathrm{s}^{-1}$ & $\mathrm{~km} \mathrm{~s}^{-1}$ & $\mathrm{~km} \mathrm{~s}^{-1}$ & $\mu \mathrm{m}$ & $\mu \mathrm{m}$ & $\mu \mathrm{m}$ & $\mathrm{km} \mathrm{s}^{-1}$ & $\mathrm{~km} \mathrm{~s}^{-1}$ & $\mathrm{~km} \mathrm{~s}^{-1}$ & Jy & Jy & Jy \\
\hline$-3.810-10 \mathrm{SE}$ & $T$ & $T$ & $T$ & $T$ & $T$ & $T$ & $T$ & $T$ & $T$ & i & i & i \\
\hline$-3.429-9 \mathrm{SE}$ & / & / & / & / & / & / & / & l & / & l & 1 & / \\
\hline$-3.048-8 \mathrm{SE}$ & / & -95 & / & / & 0.008 & l & / & 95 & / & I & $<0.001$ & / \\
\hline$-2.667-7 \mathrm{SE}$ & / & -95 & -60 & / & 0.006 & 0.006 & / & 75 & 60 & / & $<0.001$ & $<0.001$ \\
\hline$-2.286-6 \mathrm{SE}$ & 1 & -145 & -90 & 1 & 0.005 & 0.009 & 1 & 60 & 90 & 1 & $<0.001$ & 0.001 \\
\hline$-1.905-5 \mathrm{SE}$ & l & -135 & -115 & / & 0.006 & 0.008 & / & 75 & 75 & / & $<0.001$ & $<0.001$ \\
\hline$-1.524-4 \mathrm{SE}$ & 105 & -150 & -190 & 0.022 & 0.022 & 0.011 & 320 & 270 & 120 & $<0.001$ & $<0.001$ & $<0.001$ \\
\hline$-1.143-3 \mathrm{SE}$ & -60 & -220 & -240 & 0.015 & 0.015 & 0.015 & 220 & 185 & 150 & $<0.001$ & 0.002 & $<0.001$ \\
\hline$-0.762-2 \mathrm{SE}$ & -45 & -145 & -210 & 0.013 & 0.013 & 0.013 & 190 & 160 & 130 & $<0.001$ & 0.003 & 0.001 \\
\hline$-0.381-1 \mathrm{SE}$ & -100 & -70 & -190 & 0.020 & 0.020 & 0.020 & 285 & 245 & 200 & 0.004 & 0.015 & 0.006 \\
\hline $0-\mathrm{CORE}$ & -100 & 40 & -170 & 0.029 & 0.029 & 0.013 & 410 & 350 & 125 & 0.011 & 0.024 & 0.010 \\
\hline $0.3810-1 \mathrm{NW}$ & -190 & -135 & -130 & 0.014 & 0.027 & 0.014 & 200 & 325 & 140 & 0.010 & 0.018 & 0.022 \\
\hline $0.7620-2 \mathrm{NW}$ & -88 & -350 & -130 & 0.021 & 0.021 & 0.021 & 300 & 260 & 210 & 0.007 & 0.012 & 0.018 \\
\hline $1.1430-3 \mathrm{NW}$ & -590 & -620 & -515 & 0.014 & 0.014 & 0.014 & 205 & 175 & 140 & 0.001 & 0.003 & 0.003 \\
\hline $1.1430-3 \mathrm{NW} 2$ & -160 & -175 & -110 & 0.010 & 0.010 & 0.010 & 145 & 125 & 100 & $<0.001$ & 0.001 & 0.003 \\
\hline $1.5240-4 \mathrm{NW}$ & -735 & -735 & -675 & 0.028 & 0.028 & 0.028 & 397 & 340 & 279 & 0.001 & 0.004 & 0.003 \\
\hline $1.9050-5 \mathrm{NW}$ & -830 & -740 & -710 & 0.034 & 0.034 & 0.034 & 475 & 405 & 335 & $<0.001$ & 0.002 & 0.001 \\
\hline $2.2860-6 \mathrm{NW}$ & -695 & -965 & / & 0.009 & 0.016 & l & 120 & 191 & / & $<0.001$ & $<0.001$ & l \\
\hline $2.6670-7 \mathrm{NW}$ & 1 & 1 & 1 & 1 & 1 & 1 & 1 & 1 & 1 & 1 & 1 & 1 \\
\hline $3.0480-8 \mathrm{NW}$ & l & -1055 & / & / & 0.017 & / & / & 205 & / & / & $<0.001$ & / \\
\hline $3.4290-9 \mathrm{NW}$ & l & / & l & / & l & l & / & / & / & / & l & / \\
\hline $3.8100-10 \mathrm{NW}$ & l & l & / & l & / & 1 & / & l & l & / & l & l \\
\hline
\end{tabular}

\begin{tabular}{|c|c|c|c|c|c|c|c|c|c|c|c|c|}
\hline \multirow{3}{*}{$\begin{array}{c}\text { Distance to core } \\
\left({ }^{\prime \prime}\right)\end{array}$} & \multicolumn{3}{|c|}{ Radial velocity } & \multicolumn{3}{|c|}{$F W H M$} & \multicolumn{3}{|c|}{ Turbulent velocity } & \multicolumn{3}{|c|}{ Flux } \\
\hline & [ArIII]+[MgVII $]$ & [SIV] & {$[\mathrm{NeII}]$} & {$[\overline{\text { ArIII }]+[\mathrm{MgVII}]}$} & [SIV] & [NeII] & {$[$ ArIII $]+[\mathrm{MgVII}]$} & [SIV] & {$[\mathrm{NeII}]$} & [ArIII]+[MgVII] & {$[\mathrm{SIV}]$} & {$[\mathrm{NeII}]$} \\
\hline & $\mathrm{km} \mathrm{s}^{-1}$ & $\mathrm{~km} \mathrm{~s}^{-1}$ & $\mathrm{~km} \mathrm{~s}^{-1}$ & $\mu \mathrm{m}$ & $\mu \mathrm{m}$ & $\mu \mathrm{m}$ & $\mathrm{km} \mathrm{s}^{-1}$ & $\mathrm{~km} \mathrm{~s}^{-1}$ & $\mathrm{~km} \mathrm{~s}^{-1}$ & Jy & Jy & Jy \\
\hline$-3.810-10 \mathrm{SE}$ & T & T & T & T & 1 & 1 & T & $T$ & T & i & i & l \\
\hline$-3.429-9 \mathrm{SE}$ & I & / & 1 & I & / & I & i & 1 & / & i & / & I \\
\hline$-3.048-8 \mathrm{SE}$ & / & / & / & / & / & / & l & / & / & / & j & / \\
\hline$-2.667-7 \mathrm{SE}$ & / & / & I & / & / & / & / & / & / & / & / & I \\
\hline$-2.286-6 \mathrm{SE}$ & / & / & / & / & / & / & / & / & / & / & / & / \\
\hline$-1.905-5 \mathrm{SE}$ & / & 570 & l & / & 0.014 & / & / & 165 & / & / & $<0.001$ & I \\
\hline$-1.524-4 \mathrm{SE}$ & 495 & 510 & 480 & 0.010 & 0.010 & 0.022 & 150 & 125 & 222 & $<0.001$ & 0.003 & 0.001 \\
\hline$-1.143-3 \mathrm{SE}$ & 430 & 460 & 400 & 0.009 & 0.009 & 0.009 & 130 & 110 & 90 & $<0.001$ & 0.005 & 0.003 \\
\hline$-0.762-2 \mathrm{SE}$ & 350 & 380 & 355 & 0.006 & 0.006 & 0.006 & 80 & 70 & 55 & $<0.001$ & 0.003 & 0.003 \\
\hline$-0.381-1 \mathrm{SE}$ & l & / & I & l & I & / & l & l & l & l & l & l \\
\hline 0 - CORE & / & / & / & / & / & l & / & / & / & / & I & / \\
\hline $0.3810-1 \mathrm{NW}$ & / & / & / & / & / & / & / & / & / & / & / & / \\
\hline $0.7620-2 \mathrm{NW}$ & / & 285 & / & / & 0.012 & / & l & 145 & / & / & 0.006 & / \\
\hline $1.1430-3 \mathrm{NW}$ & 290 & 350 & 440 & 0.013 & 0.013 & 0.009 & 185 & 160 & 90 & $<0.001$ & 0.001 & 0.003 \\
\hline $1.5240-4 \mathrm{NW}$ & 340 & 375 & 535 & 0.014 & 0.014 & 0.014 & 195 & 170 & 140 & $<0.001$ & 0.002 & 0.001 \\
\hline $1.9050-5 \mathrm{NW}$ & 470 & 350 & 370 & 0.027 & 0.027 & 0.027 & 385 & 330 & 270 & $<0.001$ & $<0.001$ & 0.001 \\
\hline $2.2860-6 \mathrm{NW}$ & l & 540 & 335 & / & 0.015 & 0.032 & / & 180 & 320 & / & $<0.001$ & 0.002 \\
\hline $2.6670-7 \mathrm{NW}$ & / & 465 & / & / & 0.016 & / & / & 195 & / & / & $<0.001$ & / \\
\hline $3.0480-8 \mathrm{NW}$ & / & 140 & / & / & 0.013 & / & / & 150 & / & / & $<0.001$ & / \\
\hline $3.4290-9 \mathrm{NW}$ & / & / & / & / & / & / & / & / & / & / & / & / \\
\hline $3.8100-10 \mathrm{NW}$ & I & I & 95 & I & 1 & 0.004 & 1 & 1 & 40 & 1 & I & $<0.001$ \\
\hline
\end{tabular}

Description of columns: 1. Distance from the core $\left({ }^{\prime \prime}\right) ; 2$. radial velocity of the [Ar III] $+[\mathrm{Mg}$ VII] line; 3. radial velocity of the [SIV] line; 4. radial velocity of the [NeII] line; 5. FWHM of the [Ar III]+[Mg VII] line; 6. FWHM of the [SIV] line; 7. FWHM of the [NeII] line; 8 . turbulent velocity of the $[\mathrm{Ar} \mathrm{III]}]+[\mathrm{Mg} \mathrm{VII}]$ line; 9. turbulent velocity of the [SIV] line; 10 turbulent velocity of the [NeII] line; 11. flux of the [Ar III]+[Mg VII] line; 12. flux of the [SIV] line; 13. flux of the [NeII] line. 
A. Poncelet et al.: Dynamics of the ionization bicone of NGC 1068 probed in mid-infrared with VISIR, Online Material p 3
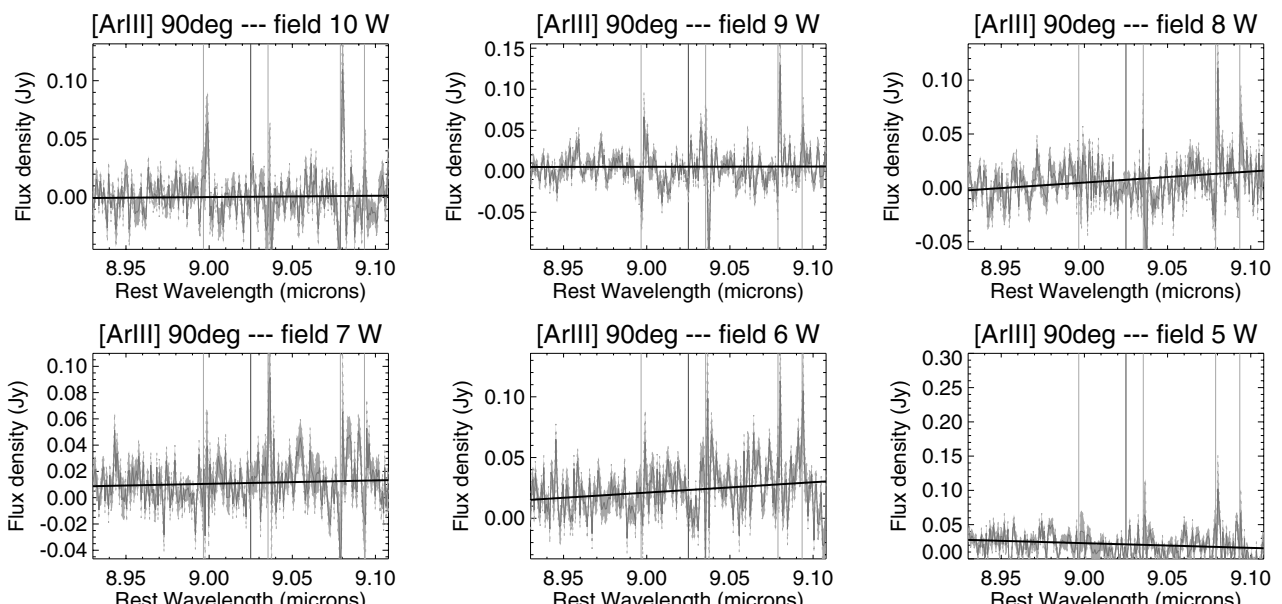

[Arlll] 90deg --- field $6 \mathrm{~W}$
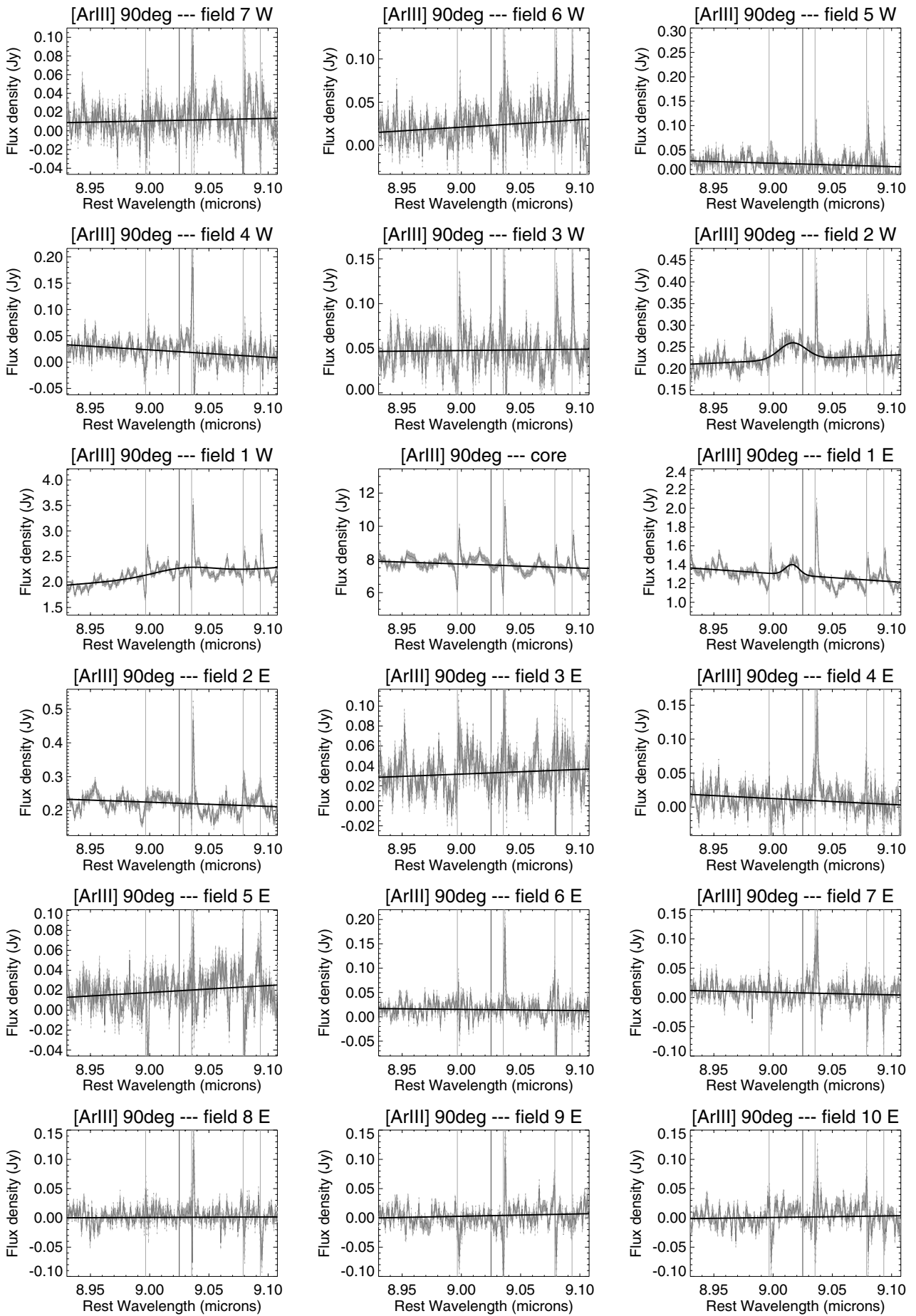

Fig. 12. Spectra in the Ar III $8.99 \mu \mathrm{m}$ filter extracted in 0.'381 steps, slit at PA $=90^{\circ}$. Reduced spectra and associated error bars are plotted in grey, and fit of the continuum plus Gaussian profiles are in black solid lines. Light and dark grey vertical lines mark the location of sky lines and of the theoretical position of the $[\mathrm{Ar} I I I]+[\mathrm{Mg}$ VII $]$ line at rest. 
A. Poncelet et al.: Dynamics of the ionization bicone of NGC 1068 probed in mid-infrared with VISIR, Online Material p 4
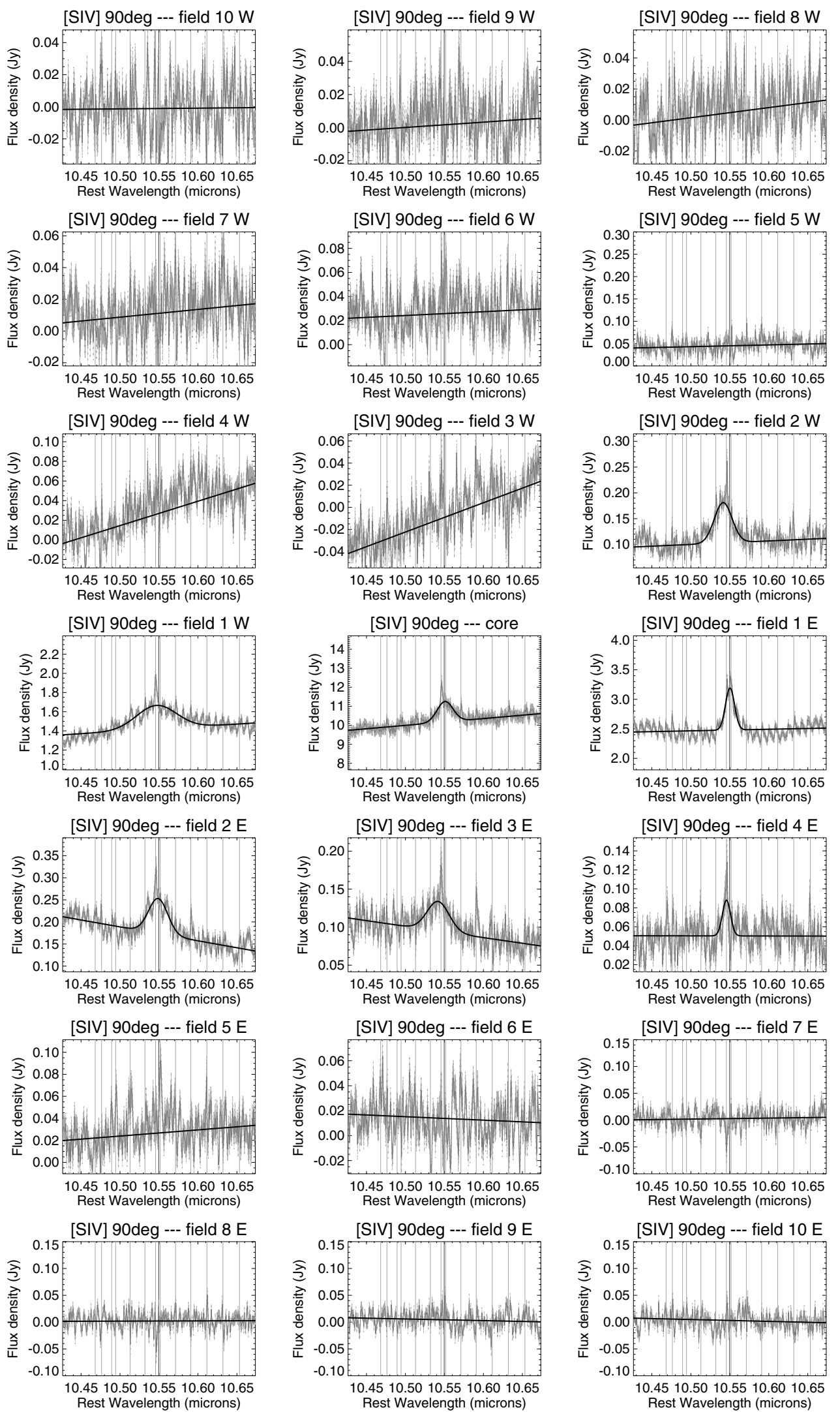

Fig. 13. Spectra in the $S I V 10.51 \mu \mathrm{m}$ filter extracted in 0.'381 steps, slit at PA $=90^{\circ}$. Reduced spectra and associated error bars are plotted in grey, and fit of the continuum plus Gaussian profiles are in black solid lines. Light and dark grey vertical lines mark the location of sky lines and of the theoretical position of the [SIV] line at rest. 
A. Poncelet et al.: Dynamics of the ionization bicone of NGC 1068 probed in mid-infrared with VISIR, Online Material p 5
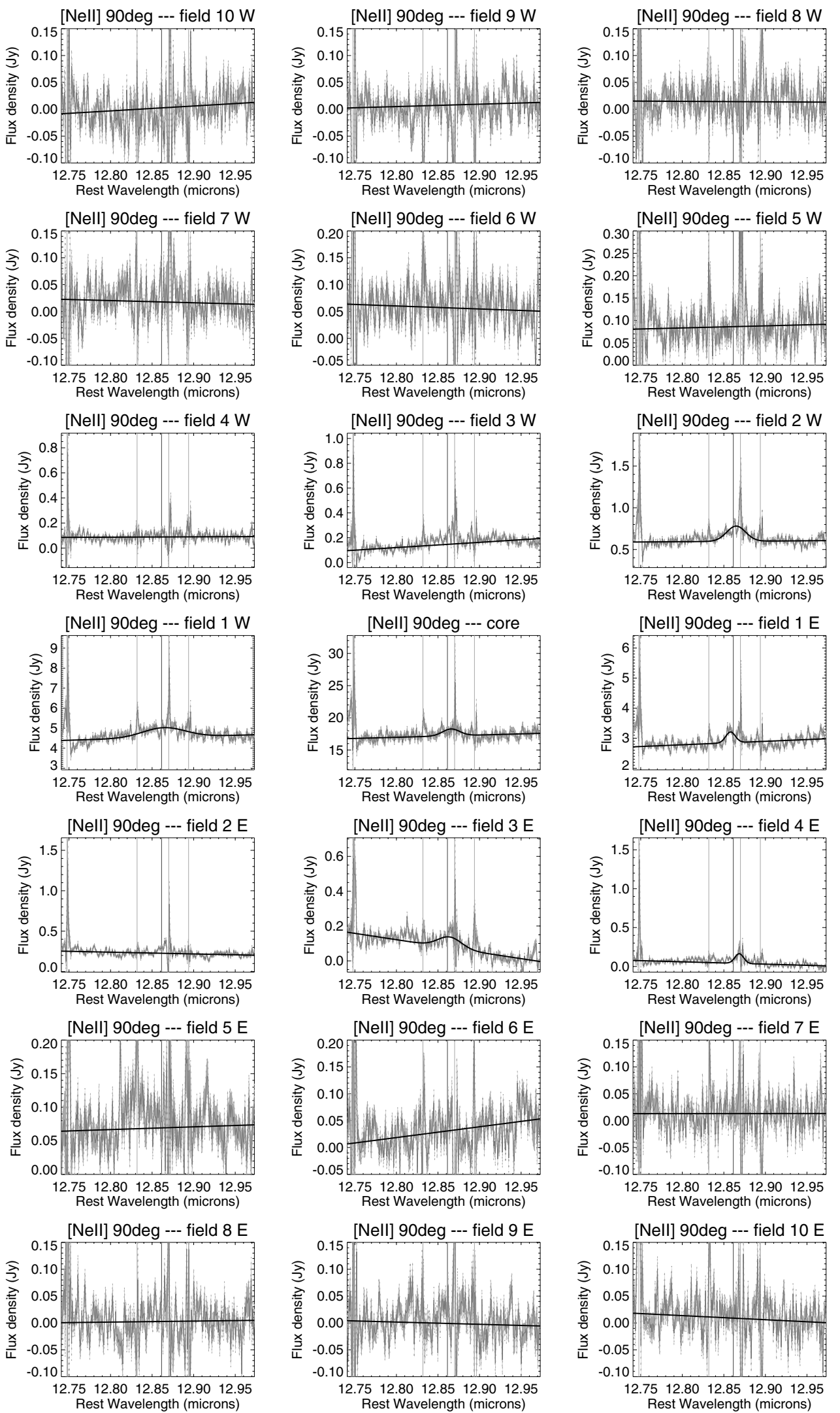

Fig. 14. Spectra in the NeII $12.81 \mu \mathrm{m}$ filter extracted in 0.'381 steps, slit at PA $=90^{\circ}$. Reduced spectra and associated error bars are plotted in grey, and fit of the continuum plus Gaussian profiles are in black solid lines. Light and dark grey vertical lines mark the location of sky lines and of the theoretical position of the [NeII] line at rest. 
A. Poncelet et al.: Dynamics of the ionization bicone of NGC 1068 probed in mid-infrared with VISIR, Online Material p 6
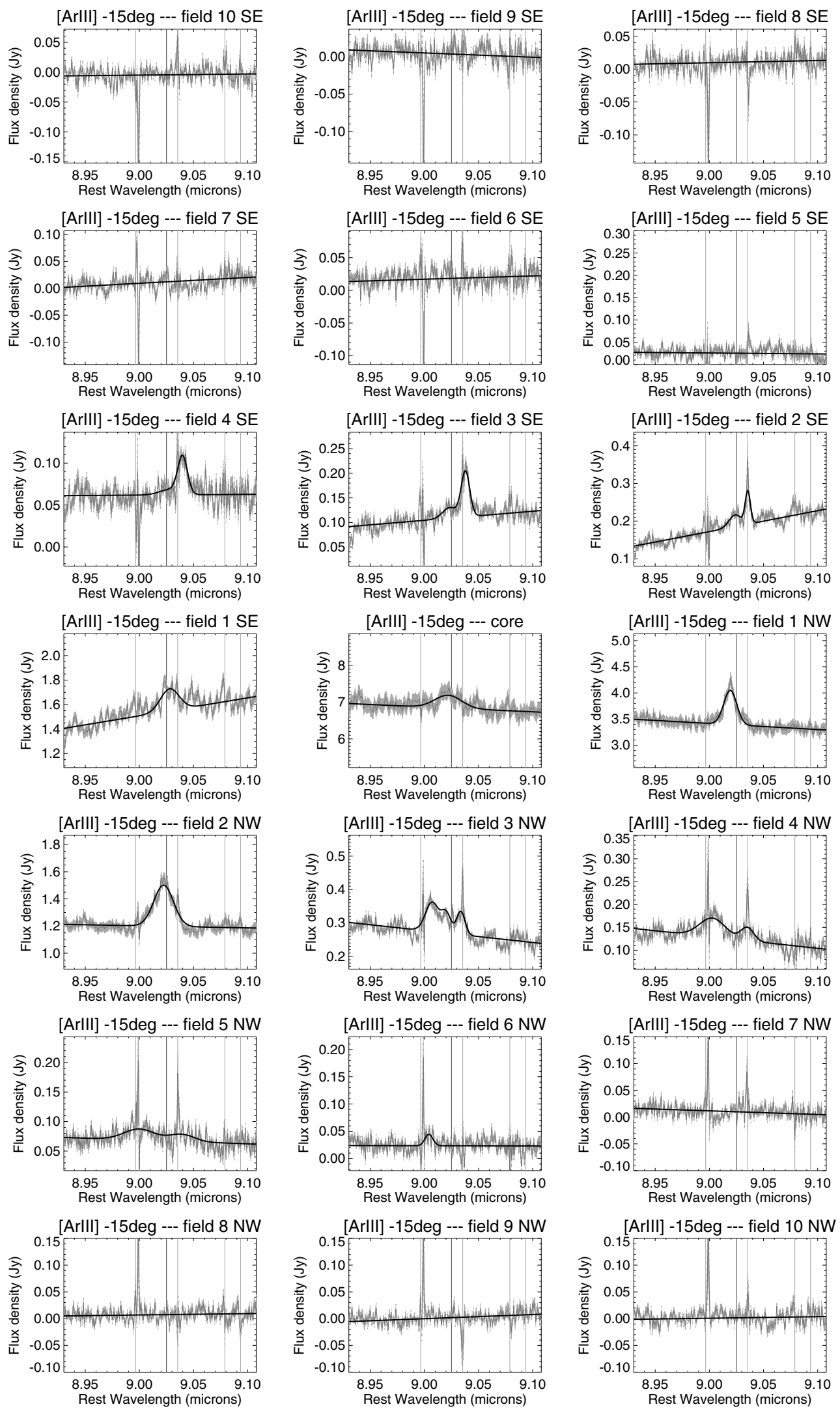

Fig. 15. Spectra of the ArIII $8.99 \mu \mathrm{m}$ blend line extracted in 0'.381 steps, slit at PA $=-15^{\circ}$. Reduced spectra and associated error bars are plotted in grey, and fit of the continuum plus Gaussian profiles are in black solid lines. Light and dark grey vertical lines mark the location of sky lines and of the theoretical position of the $[\mathrm{ArIII}]+[\mathrm{MgVII}]$ line at rest. 
A. Poncelet et al.: Dynamics of the ionization bicone of NGC 1068 probed in mid-infrared with VISIR, Online Material p 7
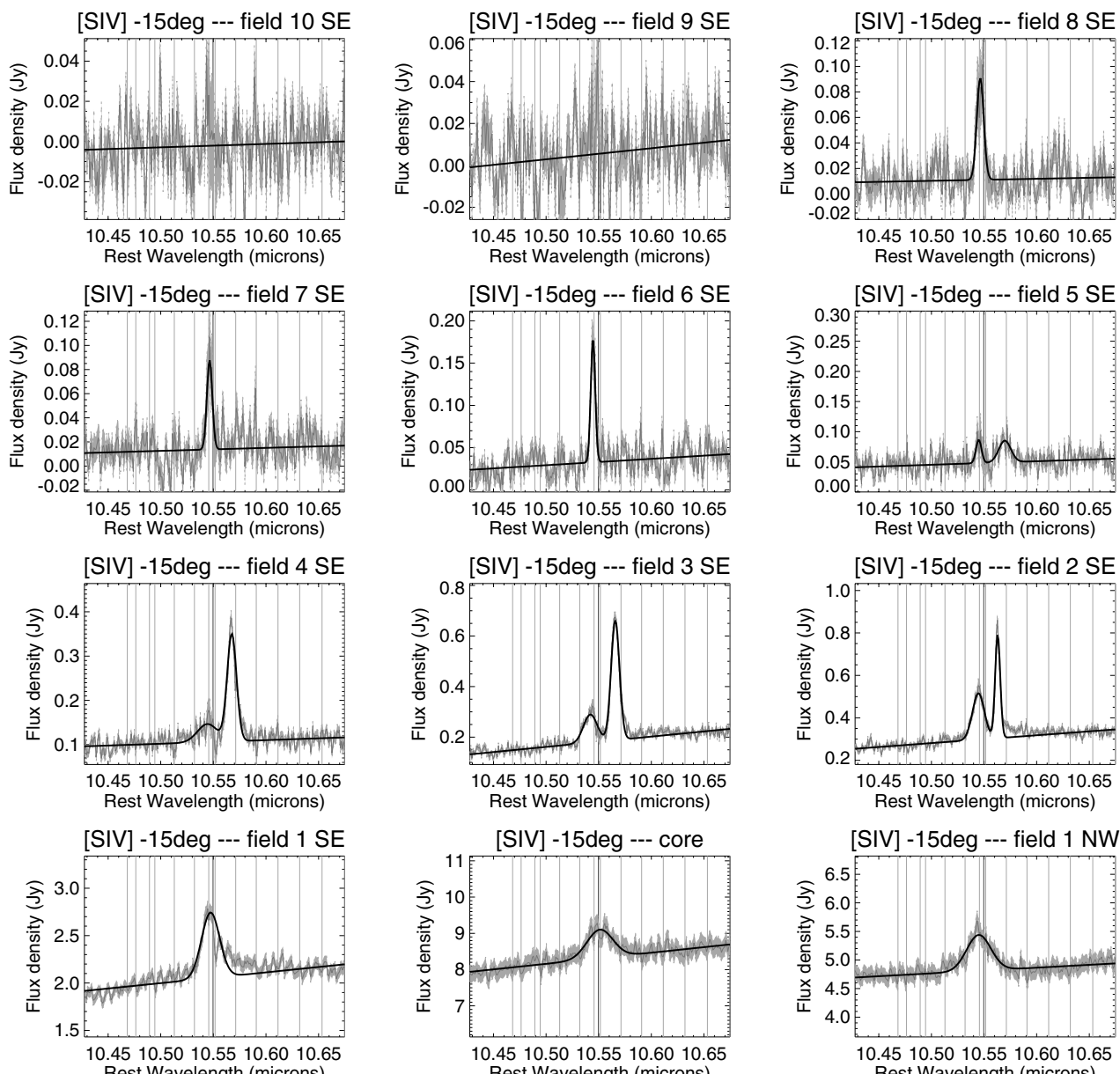

[SIV] -15deg --- field $1 \mathrm{NW}$
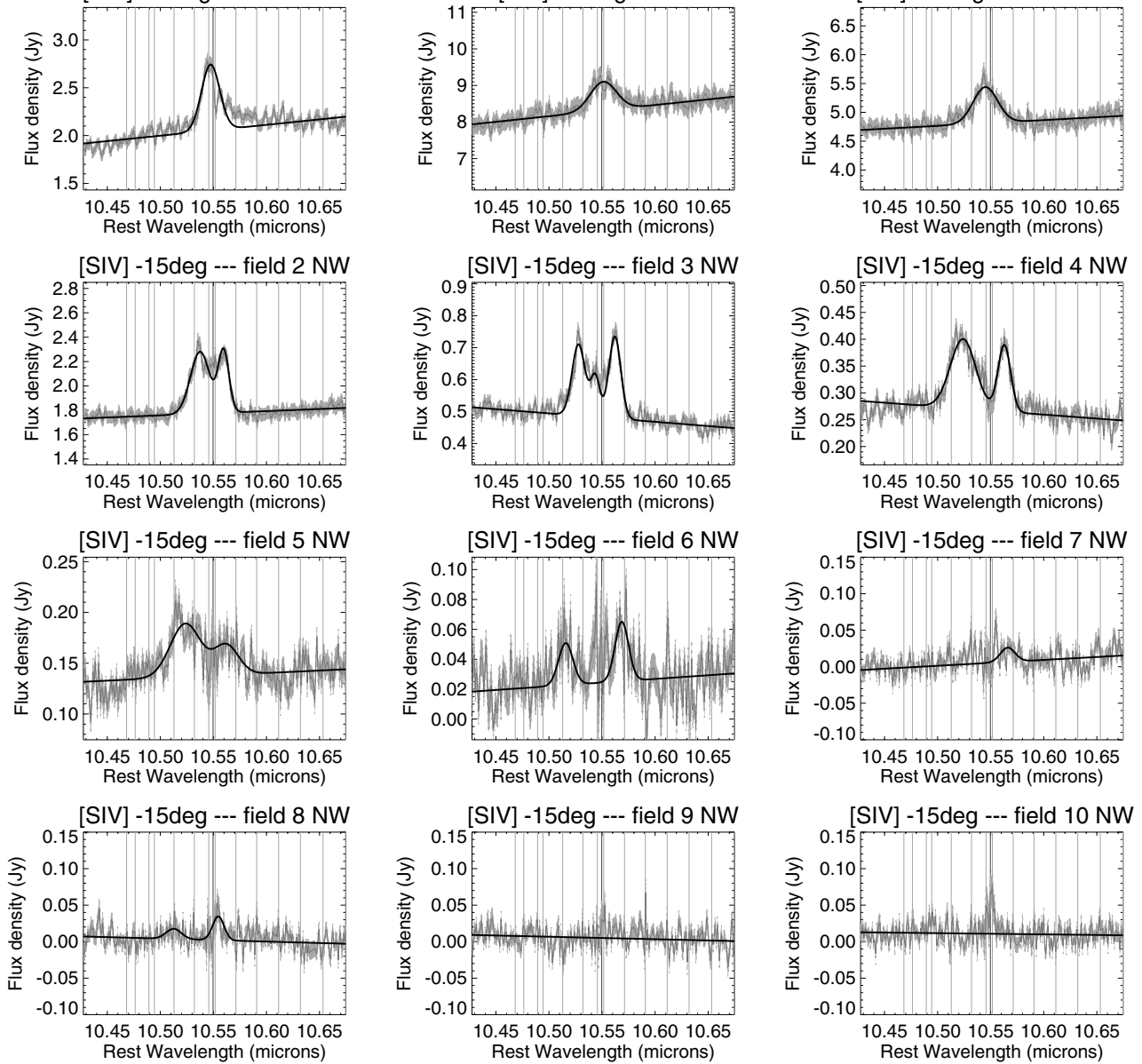

Fig. 16. Spectra of the SIV $10.51 \mu \mathrm{m}$ line extracted in 0.381 steps, slit at PA $=-15^{\circ}$. Reduced spectra and associated error bars are plotted in grey, and fit of the continuum plus Gaussian profiles are in black solid lines. Light and dark grey vertical lines mark the location of sky lines and of the theoretical position of the [SIV] line at rest. 
A. Poncelet et al.: Dynamics of the ionization bicone of NGC 1068 probed in mid-infrared with VISIR, Online Material p 8
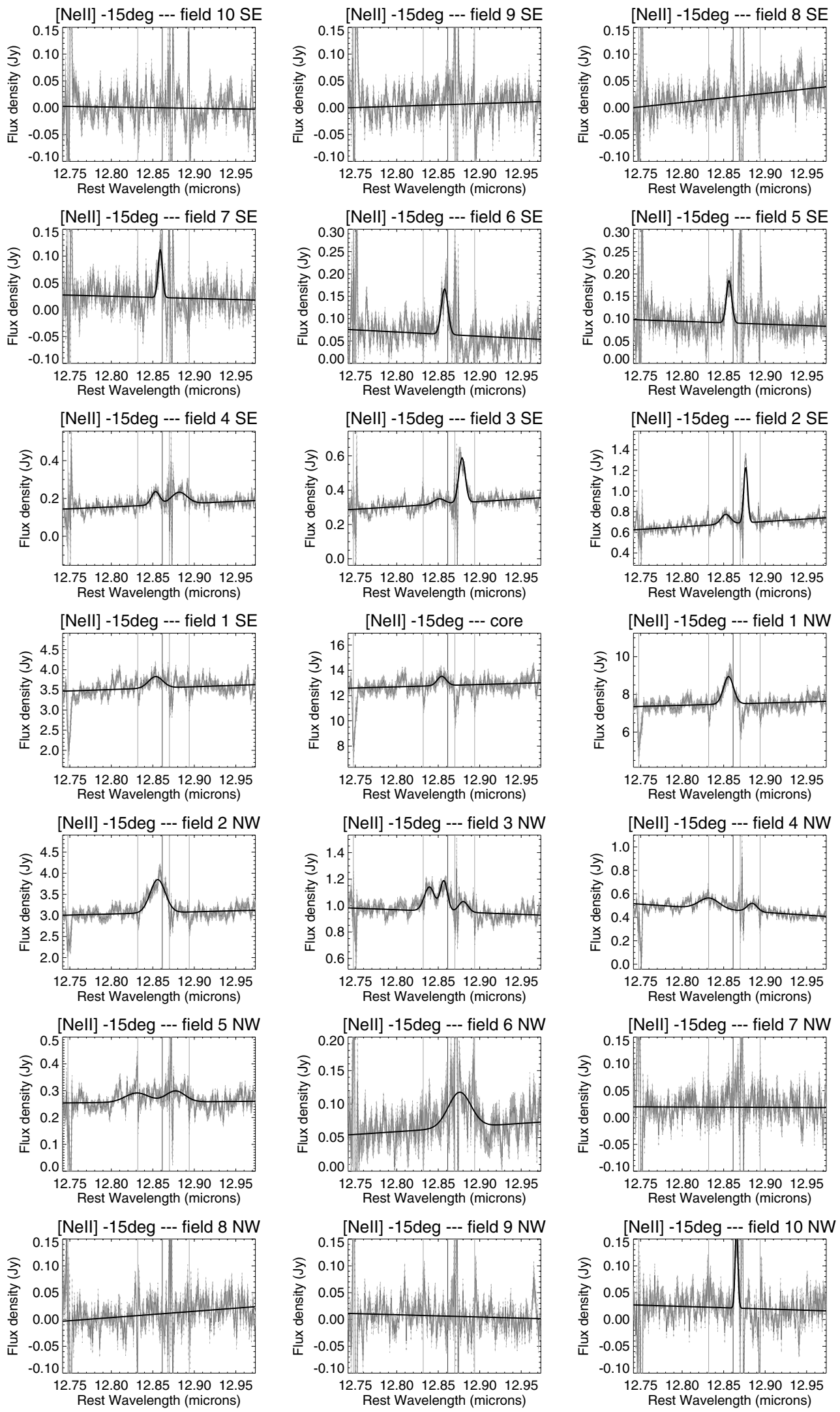

Fig. 17. Spectra of the NeII $12.81 \mu \mathrm{m}$ line extracted in 0.'381 steps, slit at PA $=-15^{\circ}$. Reduced spectra and associated error bars are plotted in grey, and fit of the continuum plus Gaussian profiles are in black solid lines. Light and dark grey vertical lines mark the location of sky lines and of the theoretical position of the [NeII] line at rest. 
A. Poncelet et al.: Dynamics of the ionization bicone of NGC 1068 probed in mid-infrared with VISIR, Online Material p 9
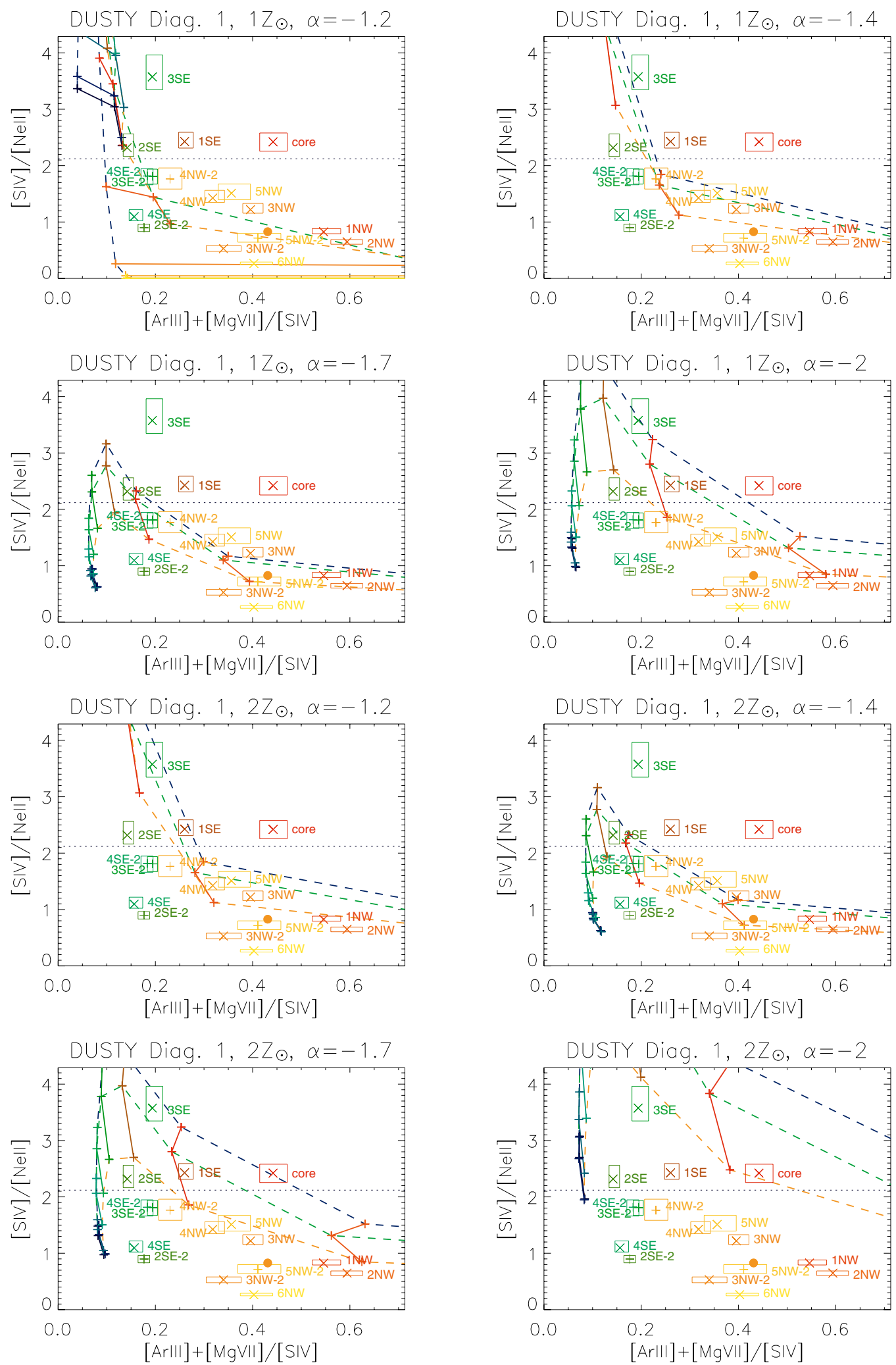

\begin{tabular}{|c|c|c|c|c|c|c|c|c|c|c|c|c|c|}
\hline density $\left(\mathrm{cm}^{\wedge}-3\right)$ & & & 100 & & & & 1000 & & & & 10000 & & \\
\hline $\log U$ & $\stackrel{0}{+}$ & -0.3 & -0.6 & $\begin{array}{c}-1.0 \\
+\end{array}$ & $\begin{array}{l}-1.3 \\
+\end{array}$ & $\begin{array}{l}-1.6 \\
+\end{array}$ & -2.0 & -2.3 & $\begin{array}{l}-2.6 \\
+\end{array}$ & $\begin{array}{r}-3.0 \\
+\end{array}$ & $\begin{array}{c}-3.3 \\
+\end{array}$ & $\begin{array}{l}-3.6 \\
+\end{array}$ & \\
\hline
\end{tabular}

Fig. 18. Line ratio grids of diagram 1: $[\mathrm{ArIII}]+[\mathrm{MgVII}] /[\mathrm{SIV}]$ vs. [SIV]/[NeII]. Ratios derived from observations are compared to predictions of DUSTY photoionization models from Groves et al. (2004b). The associated abundance values are $1 Z_{\odot}$ and $2 Z_{\odot}$, and a spectral index varying from -1.2 to -2 . Solid and dotted lines correspond to different values of the density and of the spectral index (see legend). 
A. Poncelet et al.: Dynamics of the ionization bicone of NGC 1068 probed in mid-infrared with VISIR, Online Material p 10
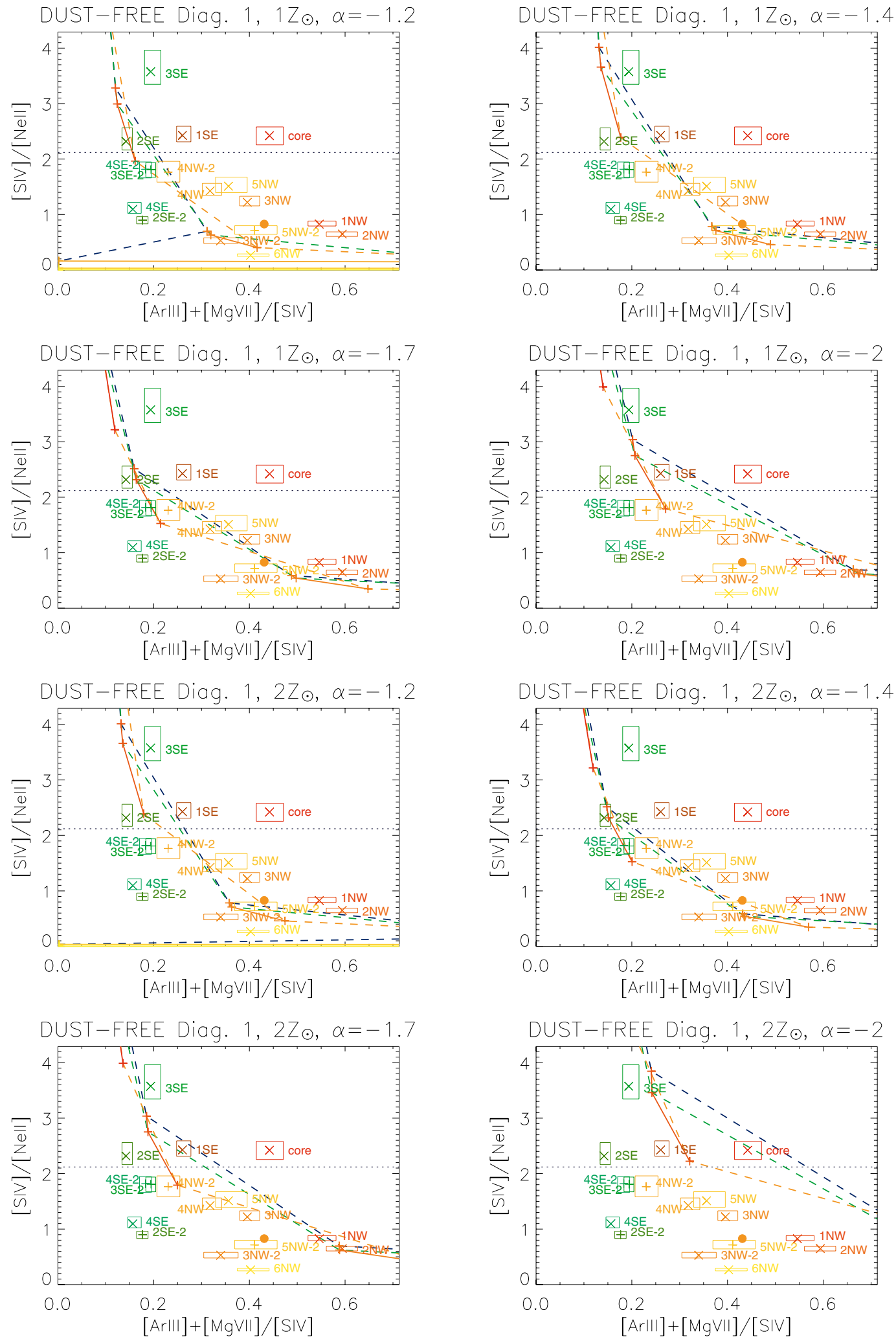

\begin{tabular}{|l|llllllllllll|}
\hline density $\left(\mathrm{cm}^{\wedge}-3\right)$ & - & & 100 & & - & - & 1000 & & $\ldots$ & 10000 \\
\hline \multirow{2}{*}{$\log \mathrm{U}$} & 0 & -0.3 & -0.6 & -1.0 & -1.3 & -1.6 & -2.0 & -2.3 & -2.6 & -3.0 & -3.3 & -3.6 \\
& + & + & + & + & + & + & + & + & + & + & + & + \\
\hline
\end{tabular}

Fig. 19. Line ratio grids of diagram 1: $[\mathrm{ArIII}]+[\mathrm{MgVII}] /[\mathrm{SIV}]$ vs. [SIV]/[NeII]. Ratios derived from observations are compared to predictions of DUST-FREE photoionization models from Groves et al. (2004b). The associated abundance values are $1 Z_{\odot}$ and $2 Z_{\odot}$, and a spectral index varying from -1.2 to -2 . Solid and dotted lines correspond to different values of the density and of the spectral index (see legend). 
A. Poncelet et al.: Dynamics of the ionization bicone of NGC 1068 probed in mid-infrared with VISIR, Online Material p 11
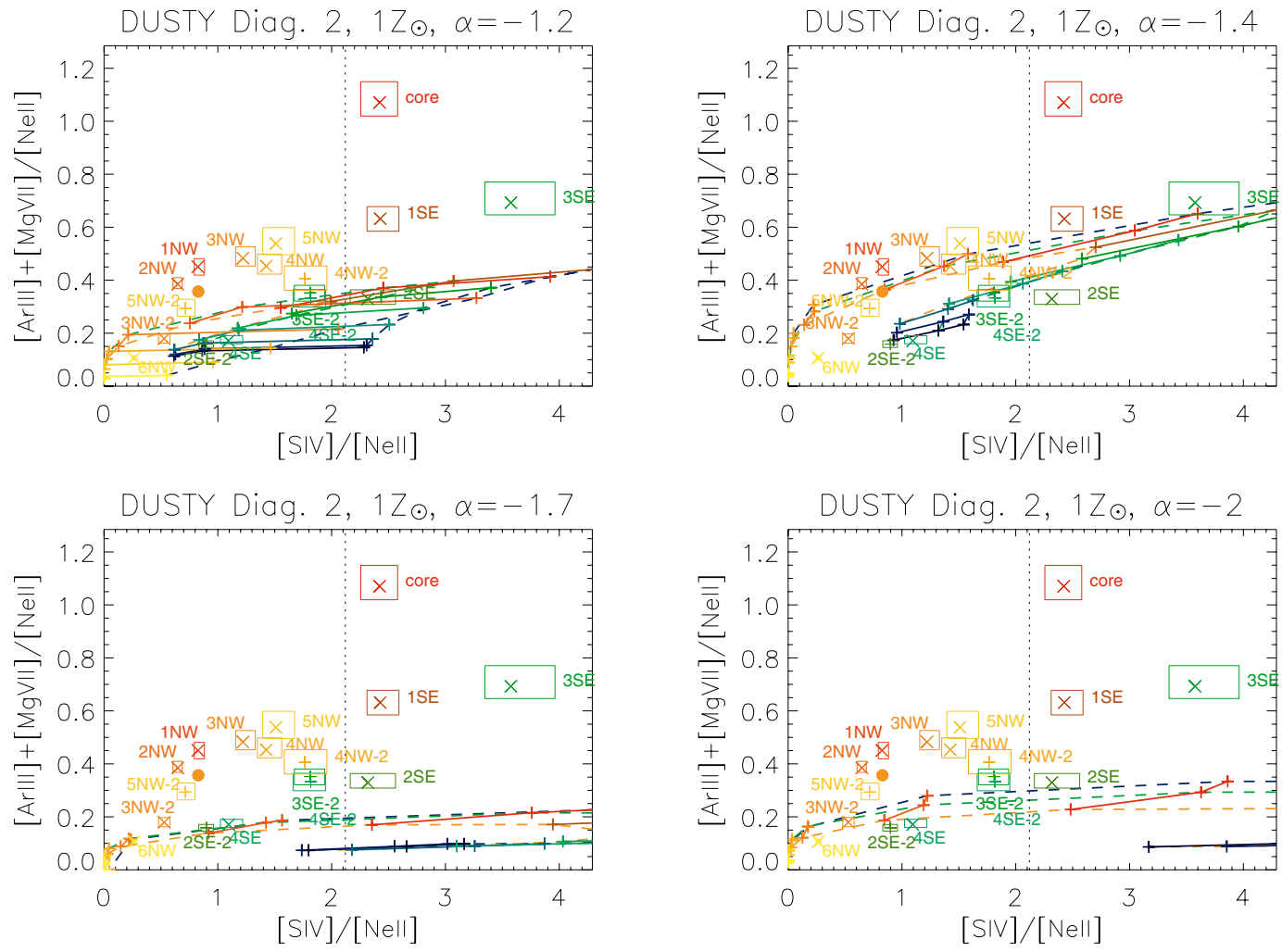

DUSTY Diag. 2, $2 Z \odot, \alpha=-1.2$
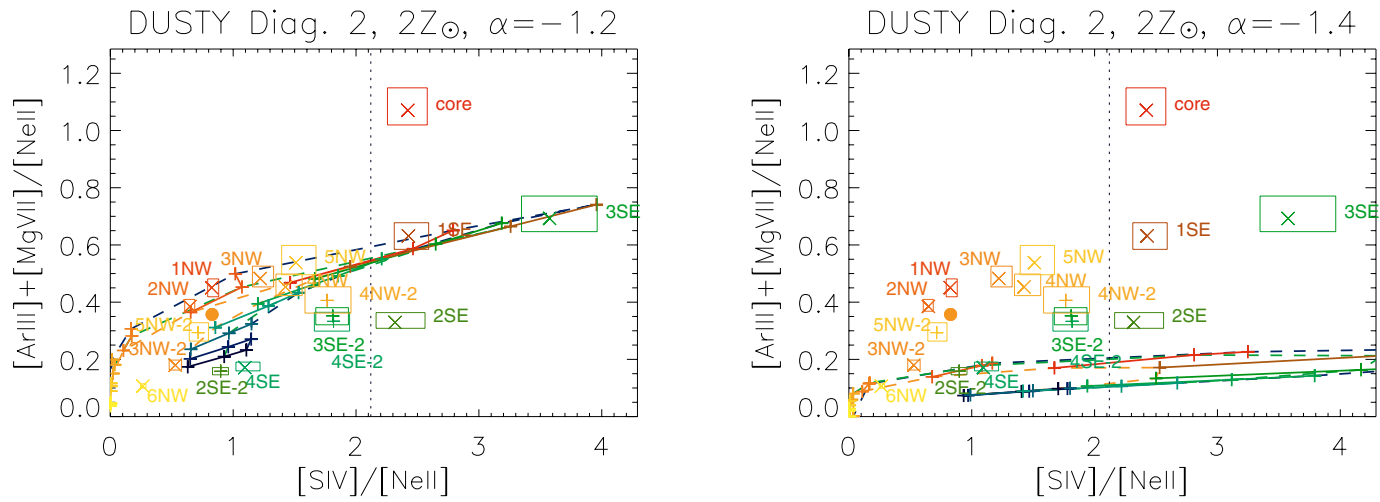

DUSTY Diag. 2, 2Z॰, $\alpha=-1.7$
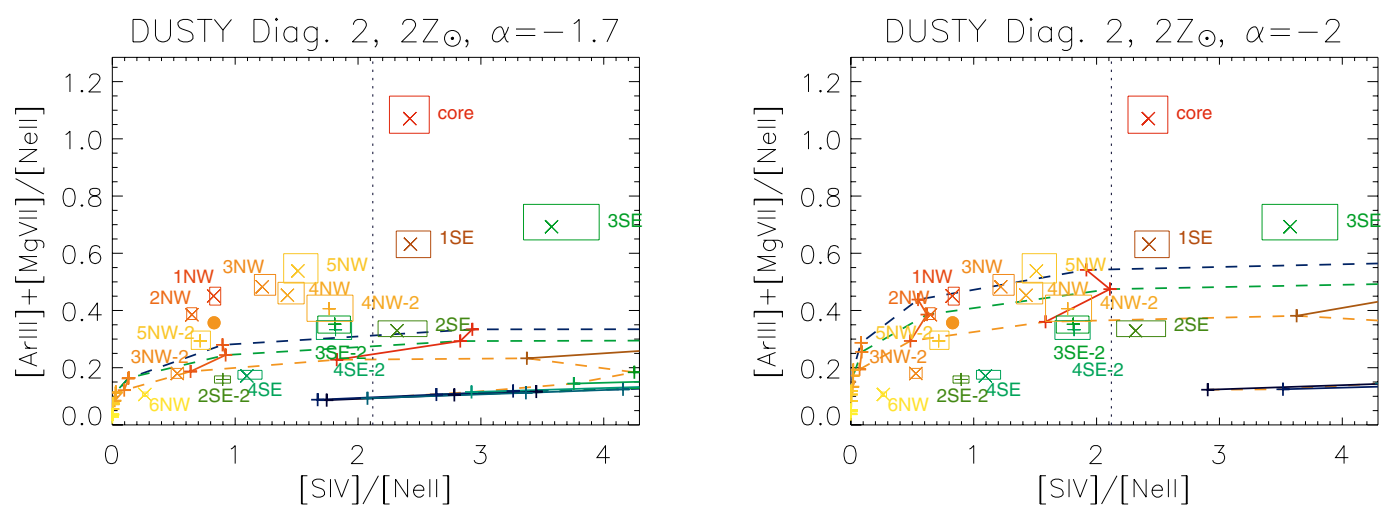

\begin{tabular}{|c|c|c|c|c|c|c|c|c|c|c|c|c|c|}
\hline density $\left(\mathrm{cm}^{\wedge}-3\right)$ & & & 100 & & & & 1000 & & & & 10000 & & \\
\hline $\log U$ & $\stackrel{0}{+}$ & -0.3 & -0.6 & $\begin{array}{c}-1.0 \\
+\end{array}$ & $\begin{array}{l}-1.3 \\
+\end{array}$ & $\begin{array}{l}-1.6 \\
+\end{array}$ & -2.0 & -2.3 & $\begin{array}{l}-2.6 \\
+\end{array}$ & $\begin{array}{r}-3.0 \\
+\end{array}$ & $\begin{array}{c}-3.3 \\
+\end{array}$ & $\begin{array}{l}-3.6 \\
+\end{array}$ & \\
\hline
\end{tabular}

Fig. 20. Line ratio grids of diagram 2: [SIV]/[NeII] vs. [ArIII]+[MgVII]/[NeII]. Ratios derived from observations are compared to predictions of DUSTY photoionization models from Groves et al. (2004b). The associated abundance values are $1 Z_{\odot}$ and $2 Z_{\odot}$, and a spectral index varying from -1.2 to -2 . Solid and dotted lines correspond to different values of the density and of the spectral index (see legend). 
A. Poncelet et al.: Dynamics of the ionization bicone of NGC 1068 probed in mid-infrared with VISIR, Online Material p 12
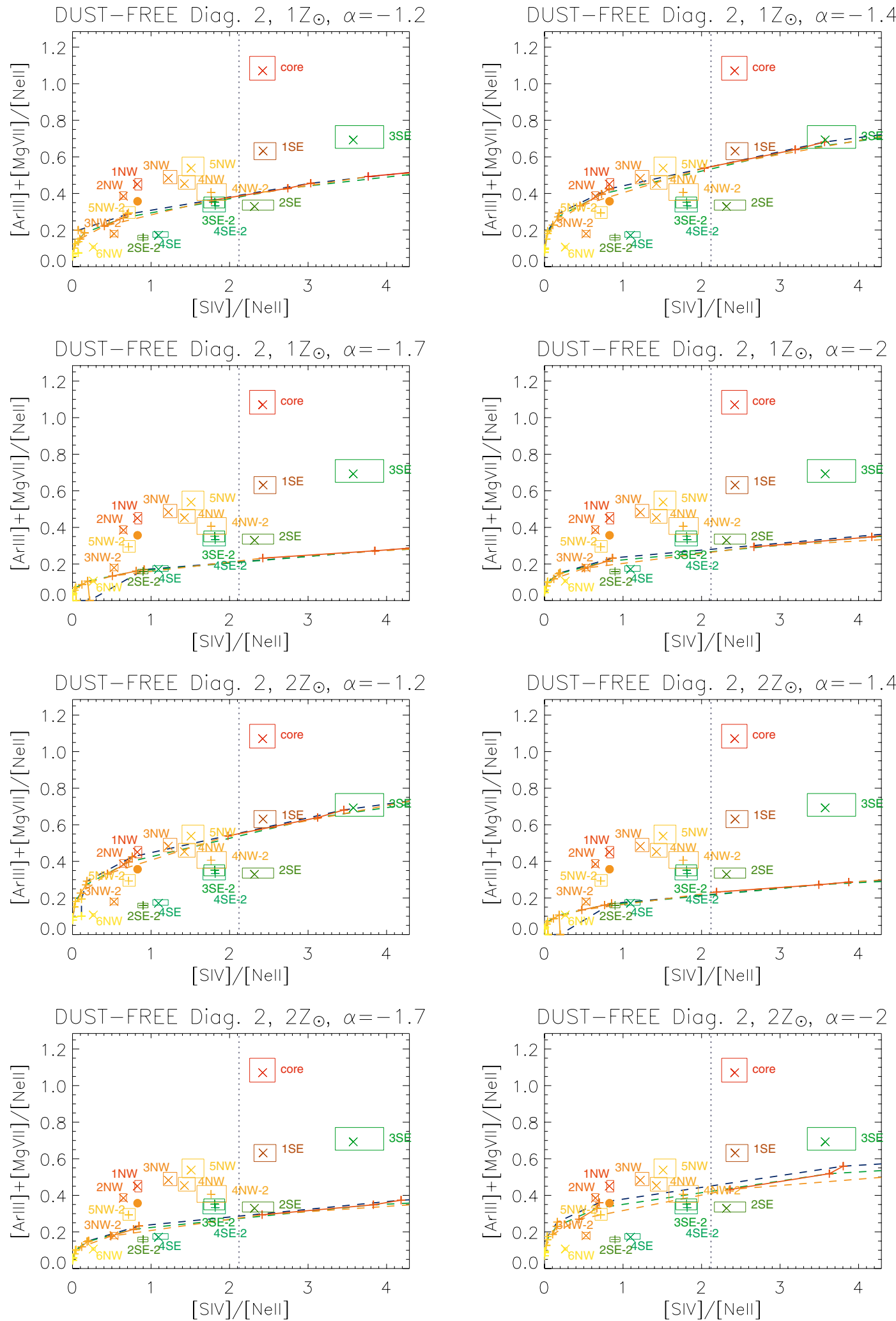

\begin{tabular}{|c|c|c|c|c|c|c|c|c|c|c|c|c|c|}
\hline density $\left(\mathrm{cm}^{\wedge}-3\right)$ & & & 100 & & & & 1000 & & & & 10000 & & \\
\hline $\log U$ & $\stackrel{0}{+}$ & -0.3 & -0.6 & $\begin{array}{r}-1.0 \\
+\end{array}$ & $\begin{array}{r}-1.3 \\
+\end{array}$ & $\begin{array}{c}-1.6 \\
+\end{array}$ & $\begin{array}{l}-2.0 \\
+\end{array}$ & -2.3 & -2.6 & $\begin{array}{l}-3.0 \\
+\end{array}$ & $\begin{array}{l}-3.3 \\
+\end{array}$ & $\begin{array}{l}-3.6 \\
+\end{array}$ & \\
\hline
\end{tabular}

Fig. 21. Line ratio grids of diagram 2: [SIV]/[NeII] vs. [ArIII]+[MgVII]/[NeII]. Ratios derived from observations are compared to predictions of DUST-FREE photoionization models from Groves et al. (2004b). The associated abundance values are $1 Z_{\odot}$ and $2 Z_{\odot}$, and a spectral index varying from -1.2 to -2 . Solid and dotted lines correspond to different values of the density and of the spectral index (see legend). 
A. Poncelet et al.: Dynamics of the ionization bicone of NGC 1068 probed in mid-infrared with VISIR, Online Material p 13
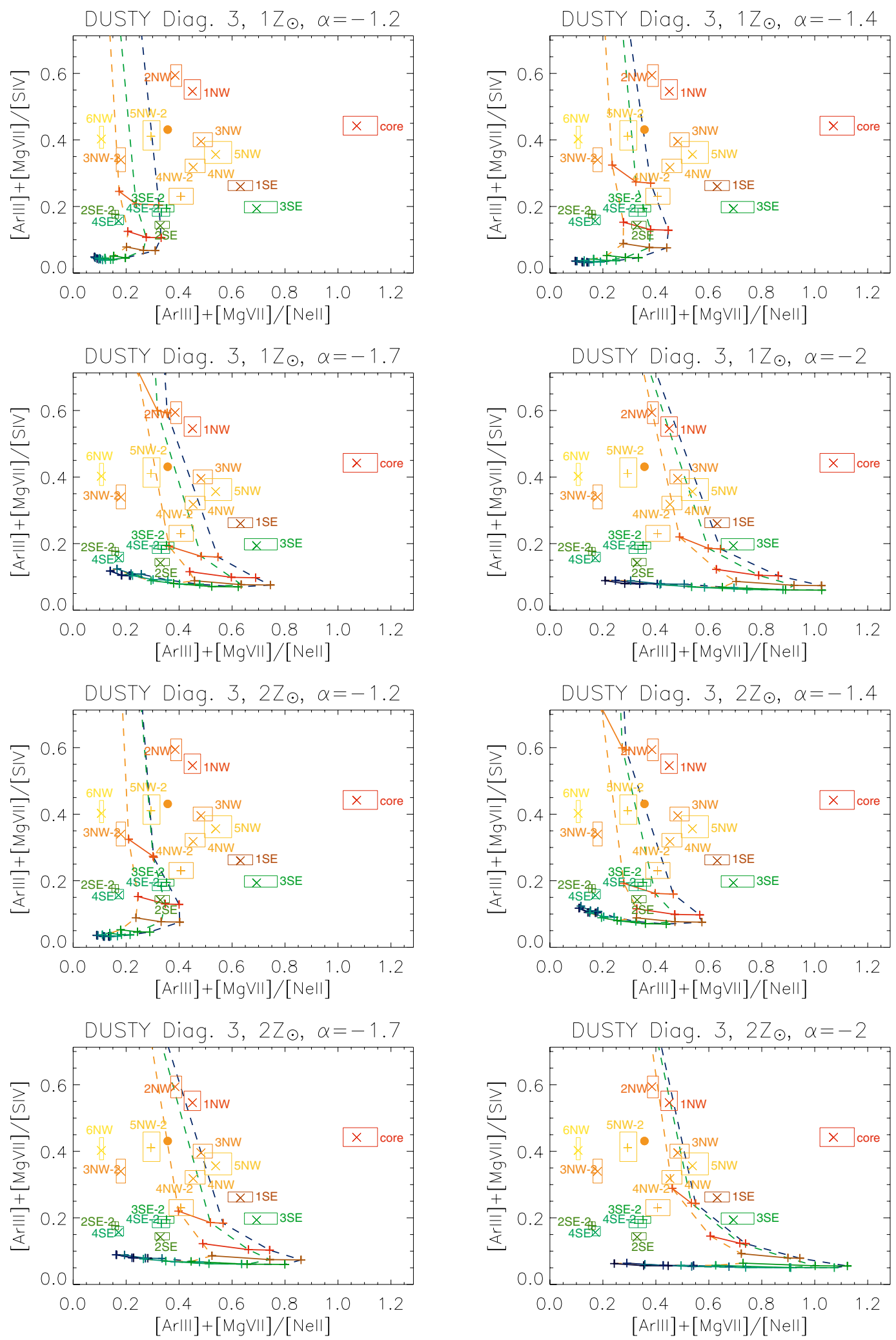

\begin{tabular}{|c|c|c|c|c|c|c|c|c|c|c|c|c|c|}
\hline density $\left(\mathrm{cm}^{\wedge}-3\right)$ & - & & 100 & & & & 1000 & & & & 10000 & & \\
\hline $\log U$ & $\begin{array}{l}0 \\
+\end{array}$ & $\begin{array}{l}-0.3 \\
+\end{array}$ & -0.6 & $\begin{array}{l}-1.0 \\
+\end{array}$ & $\begin{array}{r}-1.3 \\
+\end{array}$ & $\begin{array}{l}-1.6 \\
+\end{array}$ & $\stackrel{-2.0}{+}$ & -2.3 & -2.6 & $\begin{array}{l}-3.0 \\
+\end{array}$ & $\begin{array}{l}-3.3 \\
+\end{array}$ & $\begin{array}{l}-3.6 \\
+\end{array}$ & \\
\hline
\end{tabular}

Fig. 22. Line ratio grids of diagram 3: $[\mathrm{ArIII}]+[\mathrm{MgVII}] /[\mathrm{NeII}]$ vs. [ArIII]/[SIV] . Ratios derived from observations are compared to predictions of DUSTY photoionization models from Groves et al. (2004b). The associated abundance values are $1 Z_{\odot}$ and $2 Z_{\odot}$, and a spectral index varying from -1.2 to -2 . Solid and dotted lines correspond to different values of the density and of the spectral index (see legend). 
A. Poncelet et al.: Dynamics of the ionization bicone of NGC 1068 probed in mid-infrared with VISIR, Online Material p 14
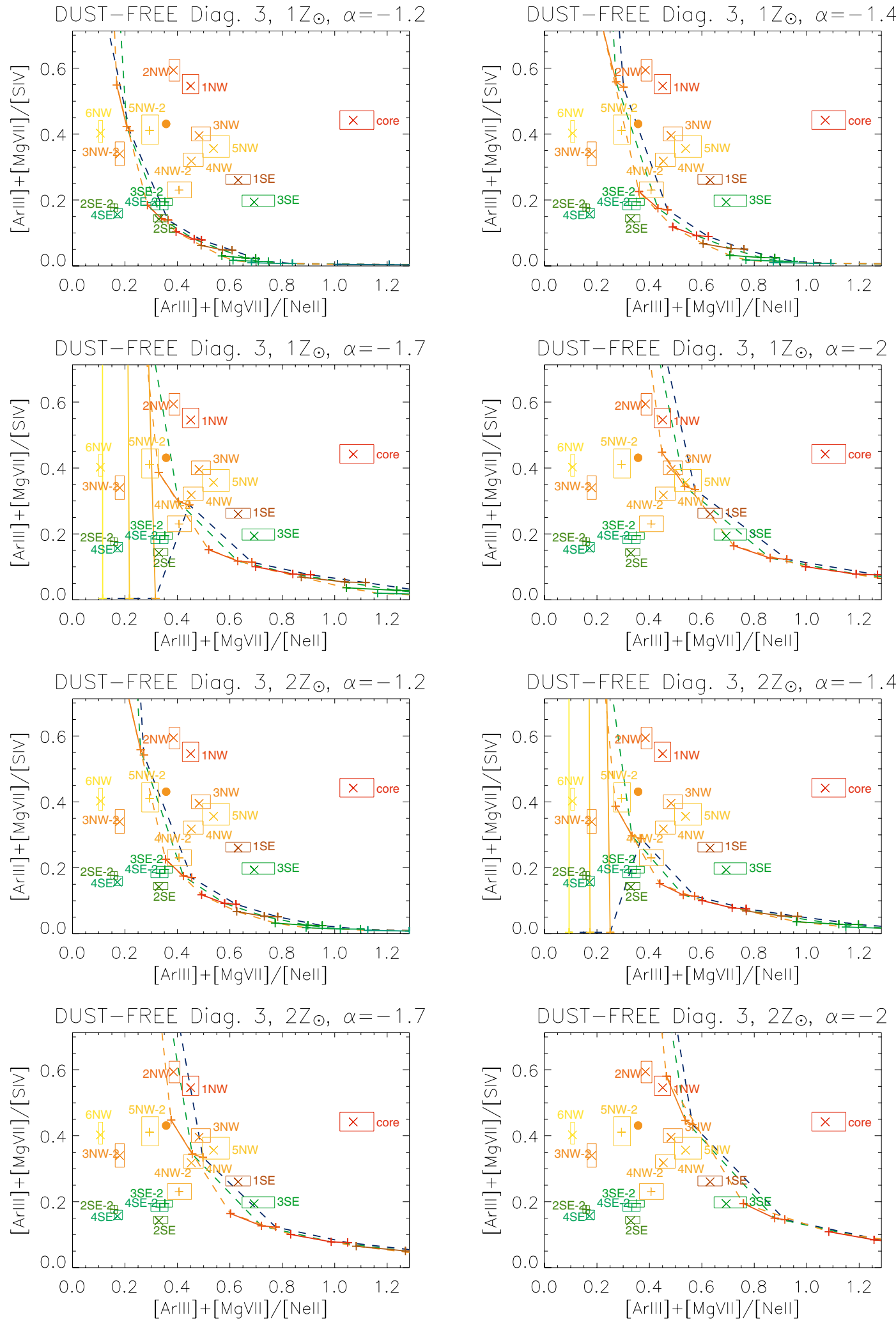

\begin{tabular}{|c|c|c|c|c|c|c|c|c|c|c|c|c|c|}
\hline density $\left(\mathrm{cm}^{\wedge}-3\right)$ & - & & 00 & & & & 1000 & & & & 10000 & & \\
\hline $\log U$ & $\begin{array}{l}0 \\
+\end{array}$ & $\stackrel{-0.3}{+}$ & $\stackrel{-0.6}{+}$ & $\begin{array}{c}-1.0 \\
+\end{array}$ & $\begin{array}{c}-1.3 \\
+\end{array}$ & $\begin{array}{l}-1.6 \\
+\end{array}$ & $\stackrel{-2.0}{+}$ & $\begin{array}{c}-2.3 \\
+\end{array}$ & $\stackrel{-2.6}{+}$ & $\begin{array}{l}-3.0 \\
+\end{array}$ & $\begin{array}{l}-3.3 \\
+\end{array}$ & $\begin{array}{c}-3.6 \\
+\end{array}$ & \\
\hline
\end{tabular}

Fig. 23. Line ratio grids of diagram 3: $[\mathrm{ArIII}]+[\mathrm{MgVII}] /[\mathrm{NeII}]$ vs. [ArIII $] /[\mathrm{SIV}]$. Ratios derived from observations are compared to predictions of DUST-FREE photoionization models from Groves et al. (2004b). The associated abundance values are $1 Z_{\odot}$ and $2 Z_{\odot}$, and a spectral index varying from -1.2 to -2 . Solid and dotted lines correspond to different values of the density and of the spectral index (see legend). 